\title{
Precipitazioni di notevole intensità a Pavia dai dati di osservatorio dal 1908 al 1957 compresi
}

\author{
G. PANNOCCHIA
}

Ricevuto il 18 Novembre 1961

Verso la fine del 1957, mentre mi trovavo ad esplicare la mia attività a Pavia nel locale osservatorio dell'I.N.G., mi fu richiesto dal Comune della città quale fosse la massima intensità attendibile nelle precipitazioni allo scopo di poter eseguire un'adatta progettazione delle fognature ancora da realizzare o da rinnovare.

Al fine di dare una risposta, la più esatta possibile, presi in esame tutti i dati disponibili in osservatorio relativi ai temporali e precipitazioni intense. I dati che allora raccolsi si riferiscono al cinquantennio 1908-1957 e furono rilevati dall'accurato esame, contemporaneo per ogni precipitazione, dei registri meteorologici, dei registri dei temporali e dei pluviogrammi. In questi ultimi lo scorrimento in senso orizzontale è di $\mathrm{mm} 35$ per una ora; avendo determinato le distanze nel senso dello scorrimento con doppio decimetro a suddivisioni di $1 / 2 \mathrm{~mm}$ e monocolo, ammettendo uno spessore medio del grafico pluviometrico non superiore ad $1 / 4 \mathrm{di} \mathrm{mm}$, l'errore, che ritengo di aver potuto compiere nella determinazione della durata della pioggia, dovrebbe essere dell'ordine del 1/4 di mm, dell'ordine cioè di \pm 4 minuti primi; per altro sono convinto che cio non possa essere avvenuto nelle precipitazioni di più breve durata, per il loro particolare aspetto effettivo. Reputo inoltre che l'esame contemporaneo dei pluviogrammi e registri abbia consentito di contenere l'errore nella determinazione dei quantitativi delle precipitazioni entro i $\pm 2 \mathrm{~mm}$.

I dati sono raccolti nella Tab. I.

Da essi non ho escluso nessun temporale anche se l'intensità della relativa precipitazione era modesta, dato che nella richiesta (se male non ricordo) si faceva esplicito riferimento ai temporali. Tornato ad esplicare la mia attività nella sede centrale dell'I.N.G. ho voluto ripren- 
dere in esame i dati allora raccolti per trarne, se possibile, qualche caratteristica elementare sebbene ciò, dato il carattere specifico delle manifestazioni temporalesche e il limitato numero di dati riferentisi solo a 50 anni, non fosse da attendere.

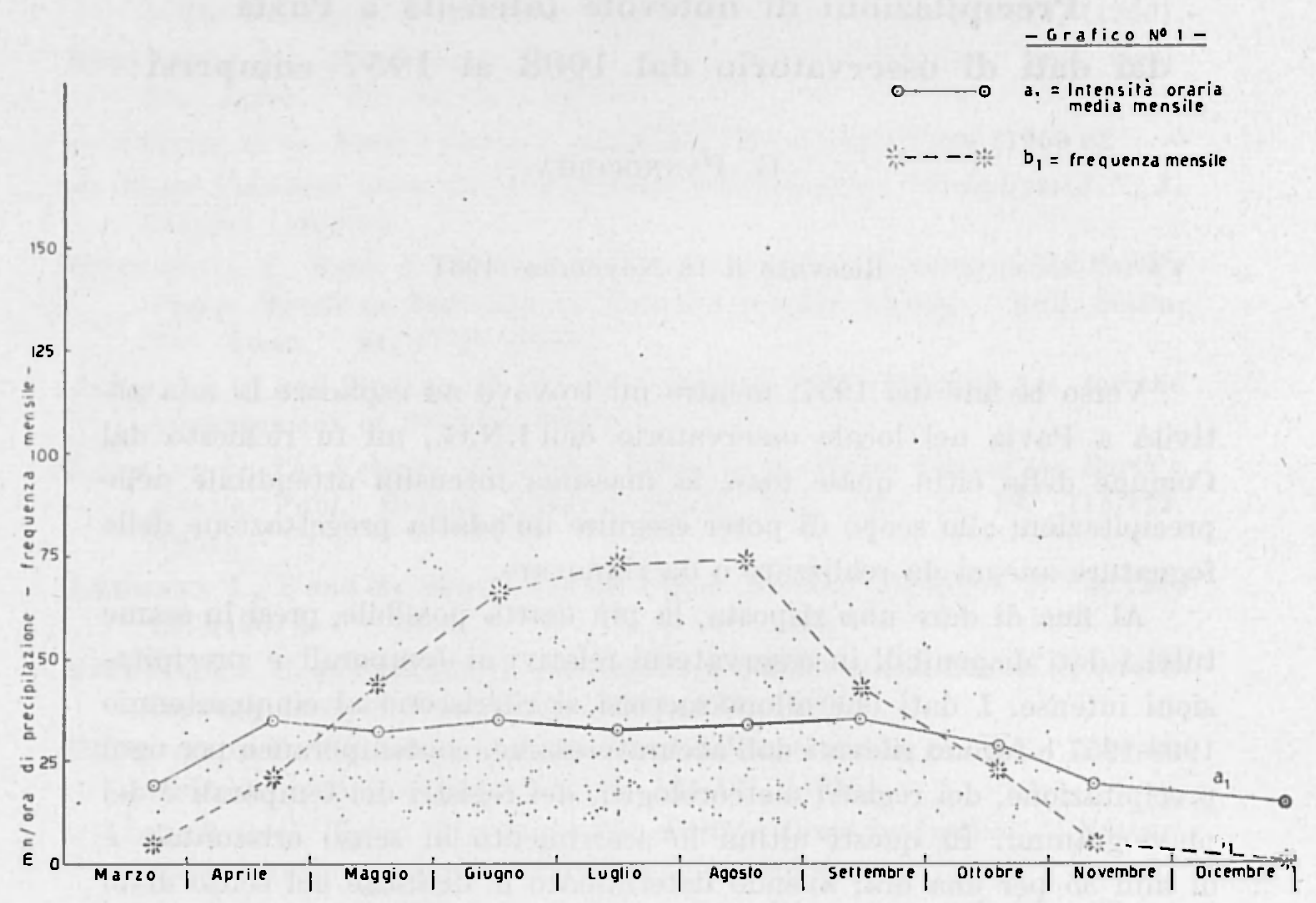

La massima intensità oraria raggiunta dalle precipitazioni si è verificata l'8-VI-1915; essa è stata di 162,0 mm/ora; la sua durata effettiva è stata anche la più breve e cioè di solo 3 minuti primi (tenuto conto del probabile errore di cui si è detto prima, questo dato potrebbe essere ritenuto incerto; in realtà non risulta tale dall'esame del pluviogramma); la massima intensità oraria con un massimo di durata effettiva di ben 162 minuti primi, si è verificata l'8-VII-1921 ed è stata di 34,3 mm/ora.

Raccogliendo i dati relativi ai cinquanta anni in Tabelle per mesi e mettendolo ciascuno alla sua data li ho riportati nel grafico n. 1: in questo le linee spezzate $a_{1}$ ) e $b_{1}$ ) riuniscono, rispettivamente i valori medi mensili delle intensità orarie di precipitazione, ed il numero di volte che tali precipitazioni si sono verificate nel mese in considerazione, in 50 anni; ambedue queste serie di punti (valori medi e frequenze) sono stati riportati sul grafico in corrispondenza al giorno medio mensile 


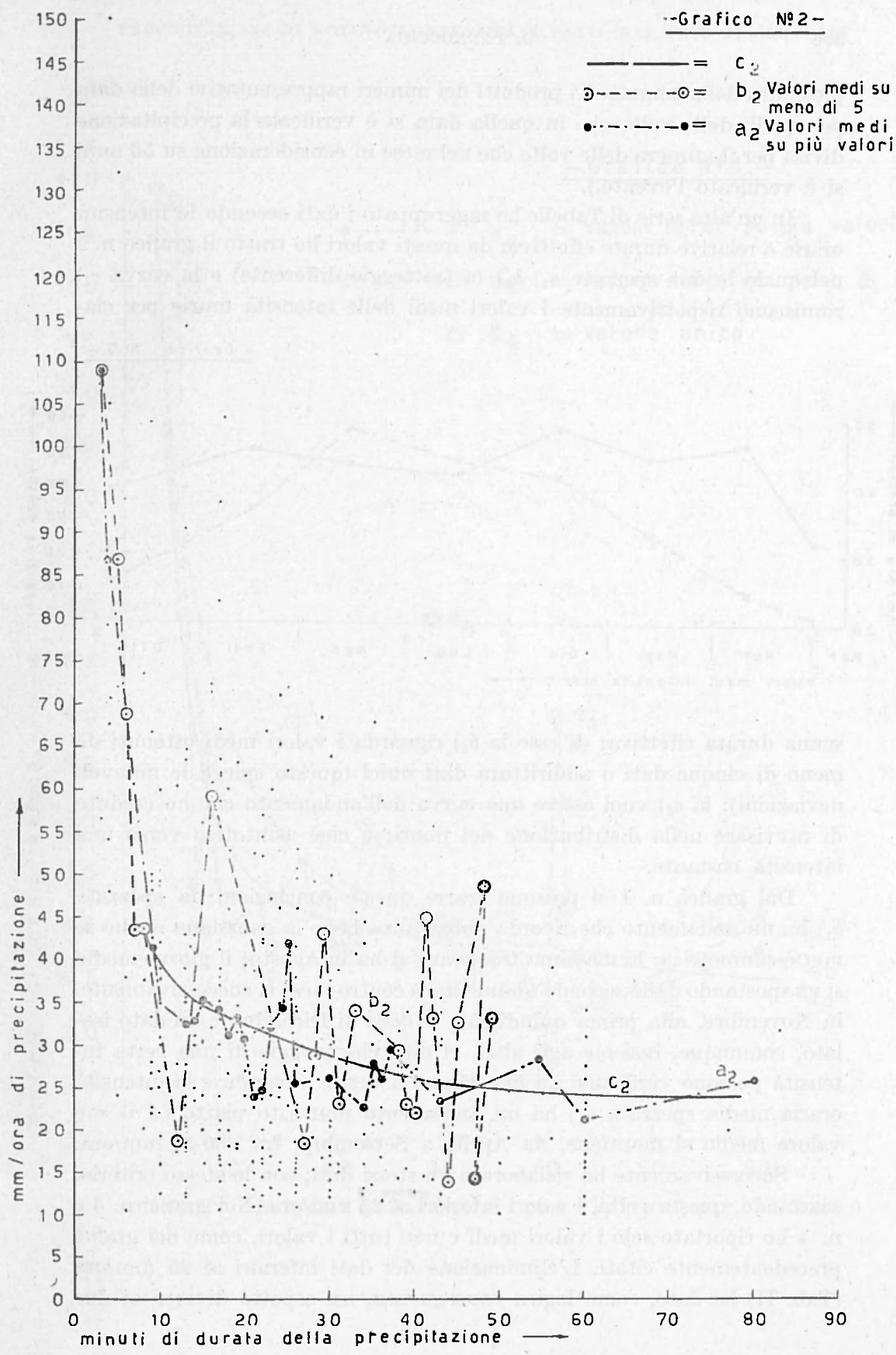


(ottenuto dalla somma dei prodotti dei numeri rappresentativi della data per quelli delle volte che in quella data si è verificata la precipitazione divisa per il numero delle volte che nel mese in considerazione su 50 anni si è verificato l'evento.).

In un'alra serie di Tabelle ho raggruppato i dati secondo le intensità orarie e relative durate effettive; da questi valori ho tratto il grafico n. 2 nel quale le due spezzate $a_{2}$ ) $b_{2}$ ) (a tratteggio differente) e la curva $c_{2}$ ) riuniscono rispettivamente $\mathrm{i}$ valori medi delle intensità orarie per cia-

- Grafico No 3 -

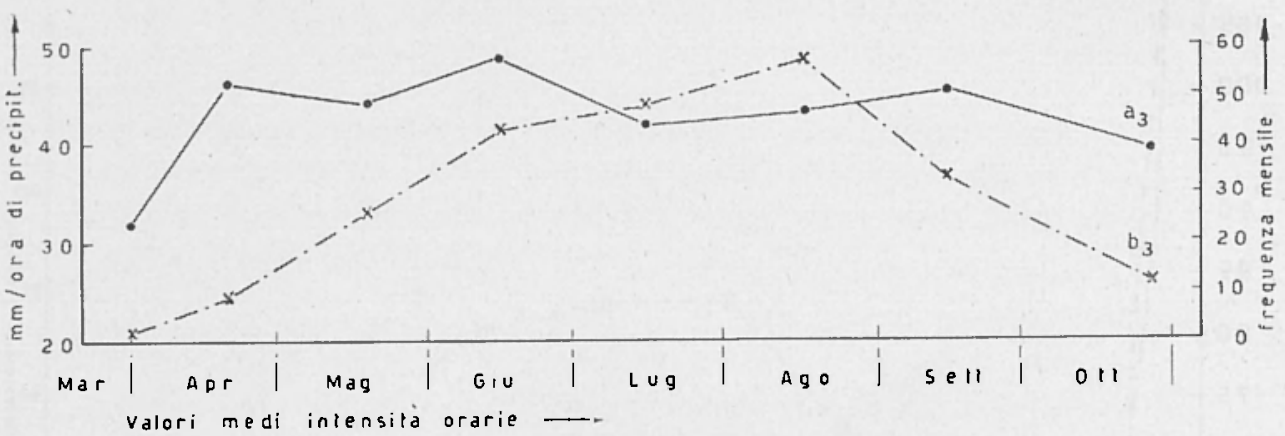

scuna durata effettiva: di esse la $b_{2}$ ) riguarda i valori medi ottenuti da meno di cinque dati o addirittura dati unici (questo spiega le notevoli deviazioni); la $c_{2}$ ) vuol essere una curva dell'andamento che ho creduto di ravvisare nella distribuzione dei punti: e ciò̀ asintotico verso una intensità costante.

Dal grafico n. 1 si possono trarre queste conclusioni: la spezzata $b_{1}$ ) ha un andamento che ricorda abbastanza bene la gaussiana anche se non è simmetrica; la massima frequenza si ha in Agosto; il giorno medio si va spostando dalla seconda quindicina a centro mese e, successivamente, in Novembre, alla prima quindicina; il dato di Dicembre è un dato isolato, comunque, insieme agli altri, ci dice che pioggie di una certa intensità possono verificarsi da fine Marzo a tutto Novembre; la intensità oraria media spezzata $a_{1}$ ) ha un andamento piuttosto piatto ed il suo valore medio si mantiene, da Aprile a Settembre, fra i $30-35 \mathrm{~mm} /$ ora.

Successivamente ho rielaborato gli stessi dati, con lo stesso criterio, scartando, questa volta, i valori inferiori ai $25 \mathrm{~mm} /$ ora. Nei grafici $\mathrm{n} .3 \mathrm{e}$ n. 4 ho riportato solo i valori medi e non tutti i valori, come nei grafici precedentemente citati. L'eliminazione dei dati inferiori ai $25 \mathrm{~mm} / \mathrm{ora}$ (Tab. II) ha dato, come logica conseguenza, un aspetto diverso ai due 
PRECIPITAZIONI DI NOTEVOLI INTENSITÀ A PAVIA DAL 1908 AL 1957397

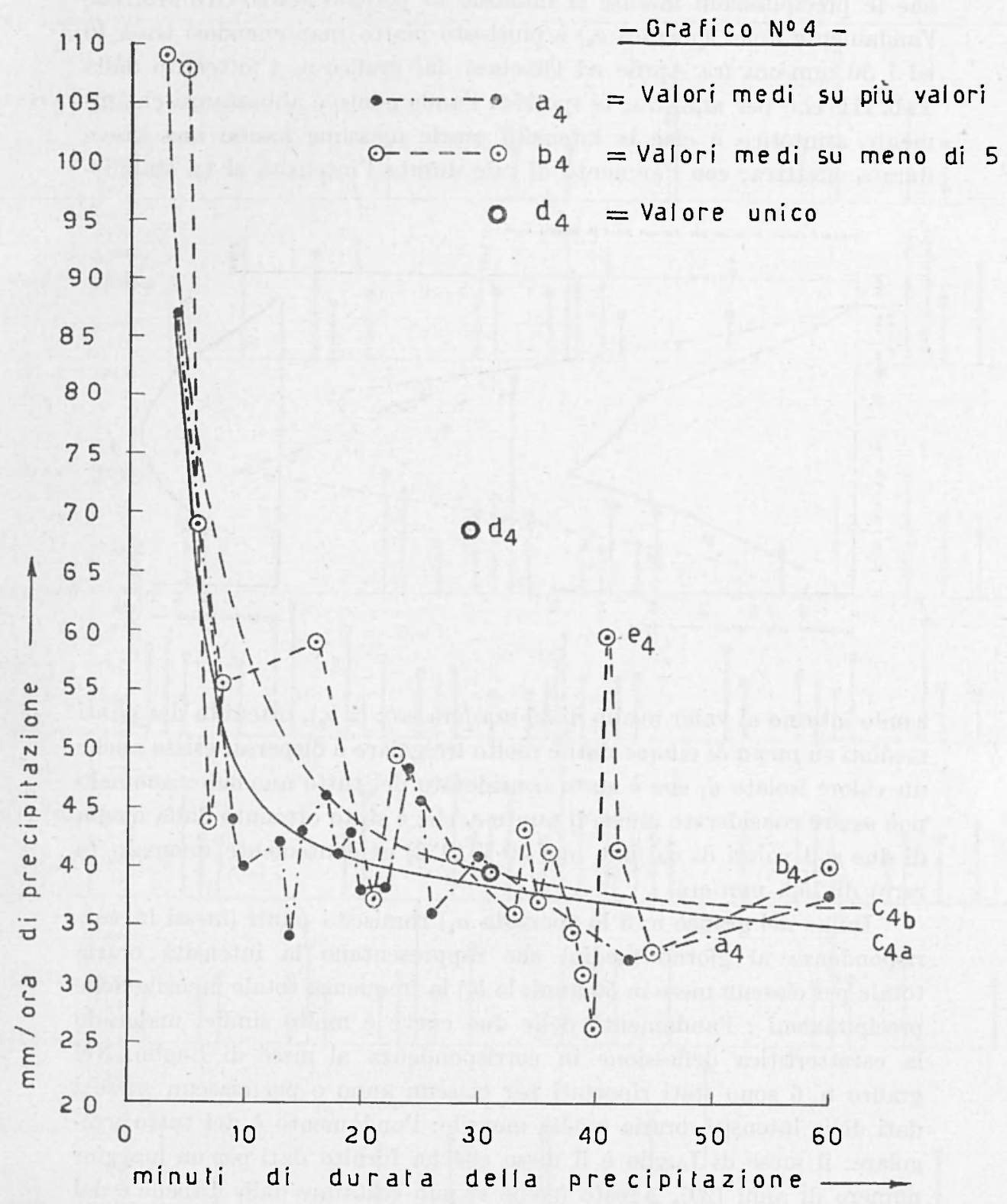


grafici; dal n. 3 è deducibile che la spezzata $b_{3}$ ) cresce gradatamente fino al massimo del mese di Agosto per poi ridiscendere più rapidamente; che le precipitazioni intense si limitano al periodo Marzo-Ottobre; che l'andamento della spezzata $a_{3}$ ) è piuttosto piatto mantenendosi tra i 40 ed i $50 \mathrm{~mm} /$ ora fra Aprile ed Ottobre; dal grafico n. 4 (ottenuto dalla Tab. III) che per ambedue le spezzate l'andamento è abbastanza chiaramente asintotico e cioè le intensità orarie massime hanno una breve durata effettiva; con l'aumento di tale durata l'intensità si va stabiliz-

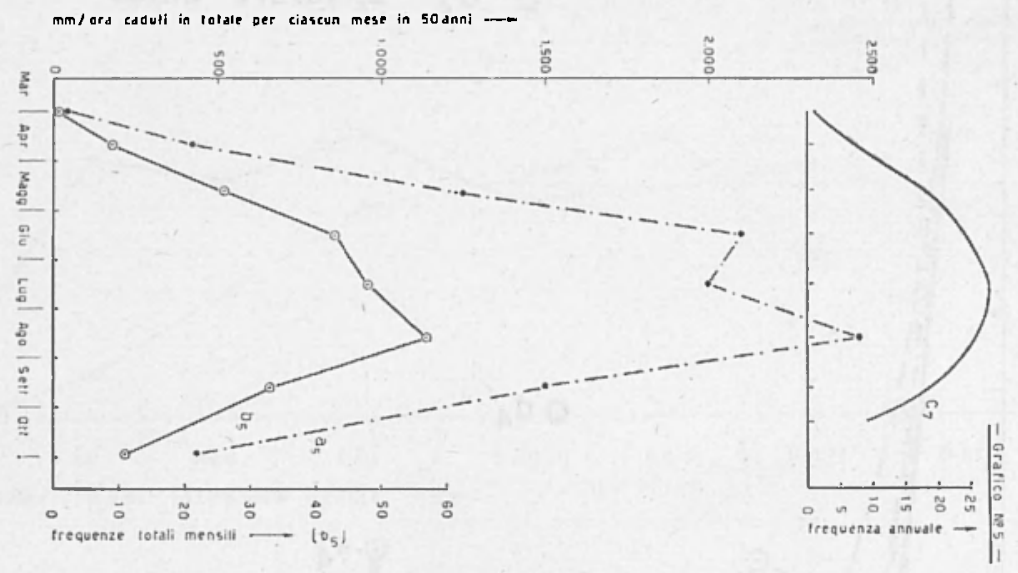

zando intorno al valor medio di $35 \mathrm{~mm} /$ ora ca.; là $b_{4}$ ), ottenuta dai punti mediati su meno di cinque dati è molto irregolare e dispersa: esiste anche un valore isolato $d_{4}$ che è stato considerato del tutto anomalo; anomalo può essere considerato anche il punto $e_{4}$ che è stato ottenuto dalla media di due soli valori di cui uno (del 30-V-1952) evidentemente anomalo (o raro) di $75,5 \mathrm{~mm} /$ ora.

Infine nel grafico $\mathrm{n} .5$ la spezzata $a_{5}$ ) riunisce i punti (messi in corrispondenza al giorno medio) che rappresentano la intensità oraria totale per ciascun mese in 50 anni; la $b_{5}$ ) la frequenza totale mensile delle precipitazioni : l'andamento delle due curve è molto simile, malgrado la caratteristica deflessione in corrispondenza al mese di Luglio. Nel grafico n. 6 sono stati riportati per ciascun anno e per ciascun mese i dati delle intensità oraria media mensile: l'andamento è del tutto irregolare: il mese di Luglio è il mese che ha fornito dati per un maggior numero di anni (29), Agosto (come si può costatare dalle Tabelle e dai grafici) quello che ha fornito il maggior numero di dati; la curva $c_{7}$ ) unisce $\mathrm{i}$ punti, messi in corrispondenza al giorno medio, che rappresen- 


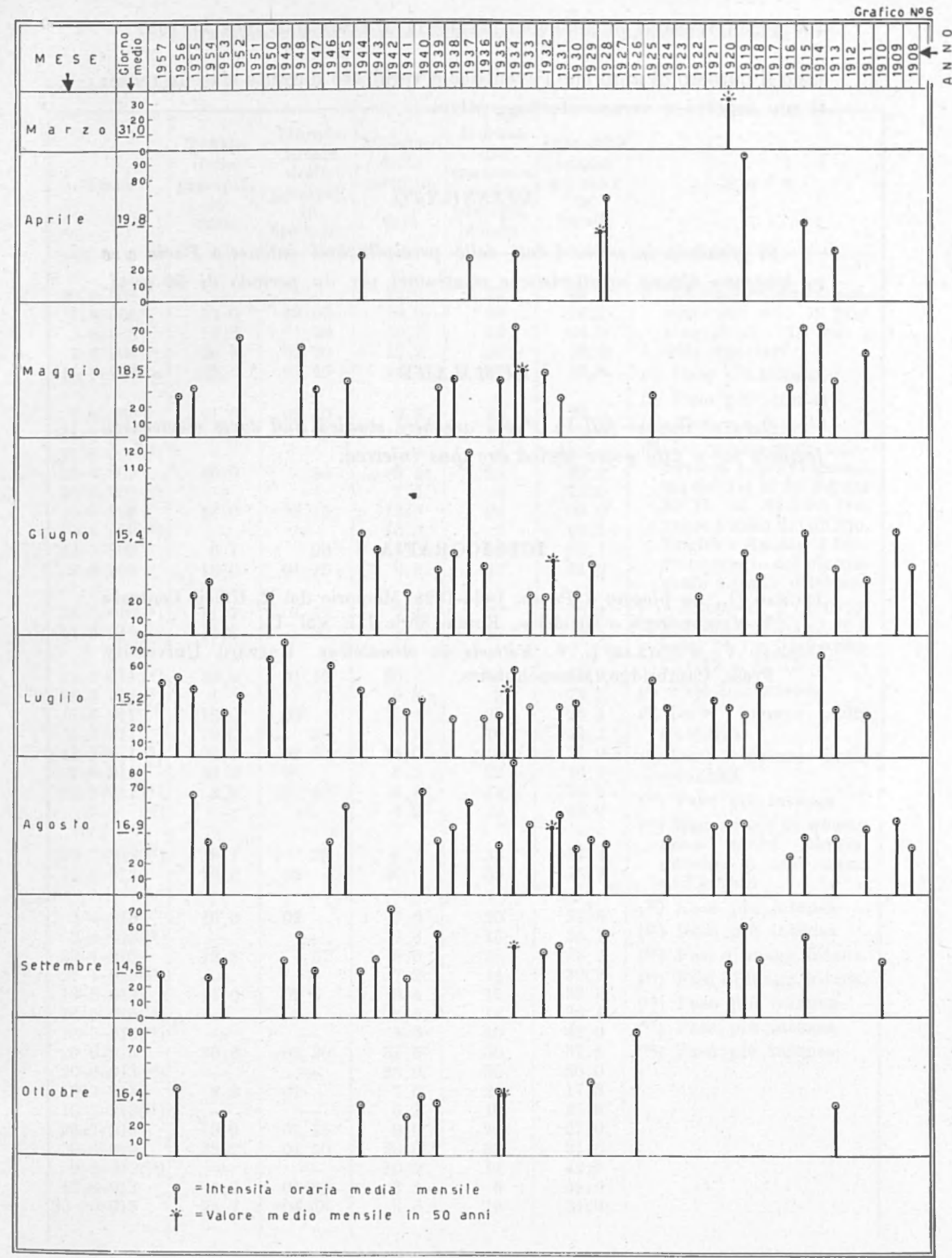


400 PRECIPITAZIONI DI NOTEVOLI INTENSITA A PAVIA DaL 1908 aL 1957

tano il numero di anni che l'evento si è verificato durante ciascun mese: il suo aspetto è veramente suggestivo.

\section{RIASSUNTO}

Si prendono in esame $i$ dati delle precipitazioni intense a Pavia e se ne traggono alcune caratteristiche elementari per un periodo di 50 anni.

\section{$S D M M A R Y$}

Data of intense fall in Pavia are here studied and some elementary features for a ffty years period are thus inferred.

\section{BIBLIOGRAFIA}

Ingrao G., La pioggia a Pavia. 1894-1928. Memorie del R. Ufficio Centrale di Meteorologia e Geoflsica, Roma, Serie III, Vol. II.

Conrad V., e Pollak L. V. Methods in climatology. Harvard University Press, Cambridge, Massachusetts. 


\begin{tabular}{|c|c|c|c|c|c|c|}
\hline Data & $\begin{array}{c}\text { Totale } \\
\text { della } \\
\text { precipit. } \\
\text { in } \\
\mathrm{mm}\end{array}$ & $\begin{array}{c}\text { Durata } \\
\text { totale } \\
\text { della } \\
\text { precipit. } \\
\text { in } \\
\text { ore min. }\end{array}$ & $\begin{array}{c}\text { Massimo } \\
\text { della } \\
\text { precipit. } \\
\text { in } \\
\mathrm{mm}\end{array}$ & $\begin{array}{c}\text { Durata } \\
\text { del } \\
\text { massimo } \\
\text { della } \\
\text { precipit. } \\
\text { in min. }\end{array}$ & $\begin{array}{c}\text { Intensità } \\
\text { oraria } \\
\text { del max } \\
\text { in } \\
\mathrm{mm} / \mathrm{h}\end{array}$ & $N o t \theta$ \\
\hline $29-6-908$ & - & - & 25,0 & 33 & 45,5 & (1) Di questo anno si pos- \\
\hline $7-8-908$ & 57,0 & 1900 & 10,0 & 36 & 16,7 & sedevano solo $18 \mathrm{plu}$ - \\
\hline $5-8-908$ & 13,2 & 20 & 5,2 & 13 & 24,0 & viogrammi, da cui $i$ \\
\hline $7-8-908$ & 26,8 & 0220 & 13,2 & 20 & 39,6 & dati riportati \\
\hline $15-8-908$ & 28,8 & 0245 & 14,3 & 30 & 28,6 & $\left({ }^{2}\right)$ Fase più intensa \\
\hline & & & & & & ${ }^{(3)}$ Fase più intensa \\
\hline $\begin{array}{r}8-6-909\left(^{(1)}\right. \\
11-6-909\end{array}$ & $\begin{array}{l}21,0 \\
10,4\end{array}$ & $\begin{array}{rl}02 & 40 \\
25\end{array}$ & $\begin{array}{r}9,2 \\
10,4\end{array}$ & $\begin{array}{l}25 \\
25\end{array}$ & $\begin{array}{l}24,0 \\
25,0\end{array}$ & (4) Fase più intensa \\
\hline $11-6-909\left(^{2}\right)$ & 10,4 & & 7,0 & 4 & $\begin{array}{r}20,0 \\
105,0\end{array}$ & (5) Di questo anno man- \\
\hline $25-6-909$ & 10,0 & 30 & 9,5 & 25 & 22,8 & cavano i pluviogram. \\
\hline $25-6-909\left(^{3}\right)$ & & & 7,5 & 6 & 75,0 & mi dal 3-1 al 25-4 e dal \\
\hline $10-8-909$ & 18,0 & $02 \quad 15$ & 13,0 & 26 & 30,0 & $21-11$ al 31-12: Du- \\
\hline $10-8-909\left({ }^{4}\right)$ & & & 10,0 & 9 & 66,7 & rante i mesi di Giugno, \\
\hline $18-9-909$ & 6,1 & 30 & 5,0 & 13 & 23,1 & Luglio $\theta$ Agosto il fun- \\
\hline $9-9-909$ & 10,0 & 0115 & 6,0 & 15 & 24,0 & $\begin{array}{l}\text { zionamento del pluvio- } \\
\text { grafo è stato difettoso }\end{array}$ \\
\hline $23-7-910\left(^{5}\right)$ & 11,7 & 0155 & 7,4 & 22 & 20,2 & $\left({ }^{6}\right)$ Mancano i pluvio- \\
\hline $14-9-910$ & 14,8 & 30 & 7,7 & 13 & 35,5 & grammi dal 2-1 al $20-3$ \\
\hline $28-5-911\left(^{8}\right)$ & 32,0 & $01 \quad 15$ & 25,7 & 43 & 35,9 & l'11-12 al 31.12 \\
\hline $28-5-911\left(^{7}\right)$ & & $01+80$ & 9,8 & 8 & 73,5 & (7) Fase più intensa \\
\hline $1-6-911$ & 18,0 & 03 & 11,4 & 26 & 26,3 & ${ }^{(8)}$ Dato incerto sulla \\
\hline $10-6-911$ & 10,0 & 30 & 8,2 & 10 & 49,2 & quantità \\
\hline $13-7-911\left(^{8}\right)$ & 33,2 & 0230 & 18,0 & 40 & 27,0 & (9) Dato incerto sulla \\
\hline $7-8-911$ & 33,2 & 01 & 5,5 & 22 & 16,1 & quantità \\
\hline $\begin{array}{c}23-8-911\left({ }^{8}\right) \\
-\quad\left({ }^{10}\right)\end{array}$ & 8,8 & 40 & $\begin{array}{l}8,4 \\
7,2\end{array}$ & $\begin{array}{r}13 \\
9\end{array}$ & $\begin{array}{l}38,8 \\
48,0\end{array}$ & (10) Fase più intensa \\
\hline $29-7$ & 9 & & 91 & & & $\begin{array}{l}\text { (1) Mancavano do questo } \\
\text { anno molti pluvio- }\end{array}$ \\
\hline $12-9-912$ & $27-2$ & 05 & 10,0 & $\begin{array}{l}38 \\
39\end{array}$ & $\begin{array}{l}14,4 \\
15,4\end{array}$ & $\underset{\text { utilizzabili }}{\text { grammi o non erano }}$ \\
\hline $\begin{array}{l}5-4-913 \\
5-4-913(12)\end{array}$ & 16,0 & 02 & $\begin{array}{l}7,6 \\
7,6\end{array}$ & $\begin{array}{l}20 \\
13\end{array}$ & $\begin{array}{l}22,8 \\
35,1\end{array}$ & $\begin{array}{l}\left({ }^{12}\right) \text { Fase più intensa } \\
\left({ }^{13}\right) \text { Fase più intensa }\end{array}$ \\
\hline $27-4-913$ & 12,3 & 57 & 9,6 & 26 & 22,2 & (14) Fasi di magg. intens. \\
\hline $27-4-913\left({ }^{13}\right)$ & & & 7,2 & 14 & 30,9 & (15) Fasi di magg. intens. \\
\hline $16-5-913$ & 15,0 & 02 & 9,4 & 17 & 33,2 & (18) Fase più intensa \\
\hline$\left|\begin{array}{|l}16-5-915(14) \\
16-5-915(15)\end{array}\right|$ & - & - & $\begin{array}{l}8,5 \\
7-0\end{array}$ & 14 & $\begin{array}{l}36,4 \\
42,0\end{array}$ & (17) Fase più intensa \\
\hline $\begin{array}{l}16-5-915\left({ }^{15}\right) \\
20,6,913\end{array}$ & $\overline{36,6}$ & $02 \overline{3} 0$ & $\begin{array}{r}7-0 \\
31,5\end{array}$ & $\begin{array}{l}10 \\
50\end{array}$ & $\begin{array}{l}42,0 \\
37,8\end{array}$ & (18) Fase più intensa \\
\hline $20-6-913\left({ }^{16}\right)$ & - & - & 25,0 & 30 & 50,0 & \\
\hline $10-7-913$ & 9,3 & 01 & 7,6 & 26 & 17,5 & \\
\hline $10-7-913\left({ }^{17}\right)$ & & + & 6,3 & 10 & 37,8 & \\
\hline $23-7-913$ & 10,9 & $01 \quad 15$ & 9,0 & 20 & 27,0 & \\
\hline $9-8-913$ & $23-2$ & 0150 & 20,0 & 38 & 31,6 & \\
\hline $9-8-913\left({ }^{18}\right)$ & - & & 10,0 & 14 & 42,9 & \\
\hline $13-8-913$ & 5,7 & 0135 & 3,8 & 6 & 38,0 & \\
\hline $30-10-913$ & 14,7 & $04 \quad 15$ & 8,5 & 16 & 31,9 & \\
\hline
\end{tabular}




\begin{tabular}{|c|c|c|c|c|c|c|}
\hline Data & $\begin{array}{c}\text { Totale } \\
\text { della } \\
\text { precipit. } \\
\text { in } \\
\mathrm{nm}\end{array}$ & $\begin{array}{c}\text { Durata } \\
\text { totale } \\
\text { della } \\
\text { precipit. } \\
\text { in } \\
\text { ore min. }\end{array}$ & $\begin{array}{c}\text { Massimo } \\
\text { della } \\
\text { precipit. } \\
\text { in } \\
\mathrm{mm}\end{array}$ & $\begin{array}{c}\text { Durata } \\
\text { del } \\
\text { massimo } \\
\text { della } \\
\text { procipit. } \\
\text { in min. }\end{array}$ & $\begin{array}{c}\text { Intensità } \\
\text { oraria } \\
\text { del max } \\
\text { in } \\
\mathrm{mm} / \mathrm{h}\end{array}$ & Not e \\
\hline $8-4-914$ & 9,1 & 43 & 6,7 & 17 & 23,6 & (10) Fase più intensa \\
\hline $24-5-914$ & 21,8 & 0230 & 19,1 & 16 & 71,6 & (20) Fase più intensa \\
\hline $21-6-914$ & 17,1 & 0215 & 8,0 & 30 & 16,0 & (21) Fase niù intensa \\
\hline $16-7-914$ & 24,8 & 0155 & 22,4 & 33 & 40,7 & $\left.{ }^{(2)}\right)$ Fase pru intensa \\
\hline $16-7-914\left({ }^{19}\right)$ & - & & 19,6 & 13 & 90,5 & $\begin{array}{l}\left.{ }^{(22}\right) \text { Fase più intensa } \\
{ }^{(23)} \text { Fase più intensa }\end{array}$ \\
\hline $21-4-915$ & 172 & 0130 & 128 & 15 & $5] 2$ & (24) Mancano altri dati \\
\hline $23-4-915$ & 26,0 & 0330 & $\begin{array}{r}1,0 \\
8,4\end{array}$ & 22 & 22,9 & ${ }^{(25)}$ opp. I fase \\
\hline $23-4-915$ & - & & 8,3 & 10 & 50,4 & ${ }^{(26)}$ II fase \\
\hline $29-5-915$ & 8,0 & 50 & 5,2 & 21 & 14,9 & \\
\hline $31-5-915$ & 6,0 & 5 & 6,0 & 5 & 72,0 & \\
\hline $8-6-915$ & 23,7 & 0330 & $12 ; 2$ & 17 & 43,0 & \\
\hline $8-6-915\left({ }^{20}\right)$ & - & & 8,1 & 3 & 162,0 & \\
\hline $19-6-915$ & 18,8 & 50 & 15,6 & 22 & 42,5 & \\
\hline $19-6-915\left({ }^{21}\right)$ & - & & 14,0 & 15 & 56,0 & \\
\hline $23-6-915$ & 17,6 & 0240 & 8,5 & 26 & 19,6 & \\
\hline $30-6-915$ & 9,0 & 13 & 9,0 & 13 & 41,5 & \\
\hline $30-6-915\left({ }^{22}\right)$ & - & - & 9,0 & 10 & 54,0 & \\
\hline $13-8-195$ & 10,7 & 0230 & 9,0 & 30 & 18,0 & \\
\hline $13-8-915$ & 18,7 & 30 & 18,7 & 30 & 37,4 & \\
\hline $22-8-915$ & 17,8 & 0100 & 14,8 & 26 & 34,2 & \\
\hline $22-8-915\left({ }^{23}\right)$ & - & & 14,4 & 20 & 43,2 & \\
\hline $4-9-915$ & 19,3 & 16 & 19,3 & 16 & 72,4 & \\
\hline $4-9-915$ & 11,5 & 0240 & 9,8 & 20 & 29,4 & \\
\hline $25-9-915$ & 30,8 & 0430 & 3,7 & 10 & 22,2 & \\
\hline $30-10-915$ & 29,1 & 1045 & 6,5 & 26 & 15,0 & \\
\hline $6-5-916$ & 11,7 & 03 & 7,0 & 13 & 32,3 & \\
\hline $26-5-916$ & 11,2 & 50 & 9,5 & 24 & 23,7 & \\
\hline $12-6-916$ & 11,8 & 0335 & 4,0 & 13 & 12,3 & \\
\hline $31-8-916$ & 23,7 & 02 & 17,0 & 40 & 25,5 & \\
\hline $5-9-916$ & 8,8 & 0145 & 5,8 & 17 & 20,5 & \\
\hline $28-9-916$ & 11,3 & 0200 & 5,1 & 17 & 18,0 & \\
\hline $5-7-917\left({ }^{24}\right)$ & 27,2 & 0300 & 14,5 & 35 & 24-9 & \\
\hline $12-9-917$ & 15,7 & 0230 & 3,2 & 10 & 19,2 & \\
\hline $7-5-918$ & 27,2 & 0300 & 8,0 & 21 & 22,8 & \\
\hline $10-6-918$ & 19,8 & - & 7,6 & 15 & 30,4 & \\
\hline $10-6-918^{{ }^{20}}$ & - & - & 8,0 & 10 & 48,0 & \\
\hline $10-6-918\left({ }^{26}\right)$ & $\overline{-}$ & - & 7,1 & 30 & 14,2 & \\
\hline $11-6-918$ & 22,5 & 06 & 4,3 & 13 & 19,8 & \\
\hline $5-7-918$ & 15,3 & & 8,1 & 10 & 48,6 & \\
\hline 2-9-918 & 8,8 & 0130 & 6,1 & 10 & 36,6 & \\
\hline $17-10-918$ & 8,6 & 35 & 6,1 & 22 & 16,6 & \\
\hline
\end{tabular}




\begin{tabular}{|c|c|c|c|c|c|c|}
\hline Data & $\begin{array}{c}\text { Totale } \\
\text { della } \\
\text { precipit. } \\
\text { in } \\
\text { mm }\end{array}$ & $\begin{array}{c}\text { Durata } \\
\text { totale } \\
\text { della } \\
\text { precipit. } \\
\text { in } \\
\text { ore min. }\end{array}$ & $\begin{array}{c}\text { Massimo } \\
\text { della } \\
\text { precipit. } \\
\text { in } \\
\text { mm }\end{array}$ & $\begin{array}{l}\text { Durata } \\
\text { del } \\
\text { massimo } \\
\text { della } \\
\text { precipit. } \\
\text { in min. }\end{array}$ & $\begin{array}{c}\text { Intensità } \\
\text { oraria } \\
\text { del max } \\
\text { in } \\
\mathrm{mm} / \mathrm{h}\end{array}$ & $N o t \theta$ \\
\hline $21-4-919$ & 13,0 & 0130 & 9,4 & 6 & 94,0 & $\left({ }^{27}\right)$ opp I fase \\
\hline $24-6-919$ & 30,5 & 0130 & 21,5 & 26 & 49,6 & (28) II fase \\
\hline $24-6-919^{(2) j}$ & - & - & 20,0 & 20 & 61,5 & (a), Iase \\
\hline $24-6-919\left({ }^{28}\right)$ & - & $\longrightarrow$ & 3,4 & 9 & 22,7 & (4) I Iaso \\
\hline $15-7-919$ & 14,0 & 0150 & 4,2 & 9 & 28,0 & $\left({ }^{30}\right)$ II fase \\
\hline $30-8-919\left({ }^{2 \theta}\right)$ & 22,6 & 0215 & 5,7 & 9 & 38,0 & (31) Catena di temporali \\
\hline $30-8-919\left({ }^{30}\right)$ & - & & 8,0 & 9 & 53,3 & di cui sono riportate \\
\hline $19-9-919$ & 18,1 & 0420 & ] ], 7 & 22 & 31,9 & le fasi più intense \\
\hline 28-9-919 & 26,4 & 0230 & 18,0 & 26 & 41,5 & (32) Se si considera come \\
\hline $28-9-919$ & - & - & 15,5 & 9 & 103,3 & unico; opp. nelle fasi \\
\hline $4-10-919$ & - & - & 7,5 & 34 & 13,2 & $\left({ }^{33}\right) I^{a}$ \\
\hline $31-3-920$ & 35,4 & 09 & 11,7 & 22 & 31,9 & $\left({ }^{34}\right) I^{a}$ \\
\hline $10-7-920$ & 13,1 & 0130 & 6,5 & 30 & 13,0 & $\left(^{\mathbf{3 5}}\right) \mathrm{III}^{\mathrm{a}}$ \\
\hline $14-7-920$ & 4,8 & 17 & 4,8 & 17 & 16,9 & (36) Come unico \\
\hline $16-7-920$ & 19,0 & 0230 & 13,0 & 26 & 30,0 & (37) Fase di max. intens. \\
\hline $17-7-920$ & 8,0 & 30 & 5,9 & 10 & 35,4 & nella fase prec. \\
\hline $11-8-920$ & 21,4 & 0225 & 18,0 & 23 & 47,0 & (38) \\
\hline $19-8-920$ & 15,4 & $01 \quad 15$ & 13,0 & 17 & 45,9 & $\left.{ }^{38}\right)$ mancano altri dati \\
\hline $21-8-920$ & 28,6 & 0110 & 28,0 & 38 & 44,2 & ${ }^{\left({ }^{39}\right)}$ Mesi estivi piuttosto \\
\hline $23-9-920\left({ }^{31}\right)$ & 83,8 & 1110 & 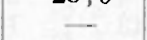 & - & - & secchi; mancano altri \\
\hline $23-9-920$ & - & - & 6,0 & 13 & 27,7 & dati. \\
\hline 23-9-920 & - & - & 22,0 & 30 & 44,0 & \\
\hline 23-9-920 & - & - & 18,0 & 39 & 27,7 & \\
\hline $7-7-921$ & 30,0 & 0150 & 27,0 & 49 & 33,1 & \\
\hline $8-7-921\left({ }^{32}\right)$ & 102,2 & 0638 & 92,7 & 162 & 34,3 & \\
\hline $8-7-921\left({ }^{33}\right)$ & - & - & 30,0 & 41 & 43,9 & \\
\hline $8-7-921\left(^{34}\right)$ & - & 一 & 39,2 & 42 & 56,0 & \\
\hline $8-7-921\left({ }^{35}\right)$ & - & - & 21,0 & 39 & 33,8 & \\
\hline $18-7-921$ & 23,0 & 57 & 22,0 & 43 & 30,7 & \\
\hline $25-7-921$ & 11,5 & 50 & 10,5 & 22 & 28,6 & \\
\hline $24-8-921\left({ }^{36}\right)$ & 50,0 & 0230 & $\longrightarrow$ & - & 20,0 & \\
\hline $24-8-921$ & - & - & 32,0 & 45 & 32,0 & \\
\hline $24-8-921\left({ }^{37}\right)$ & - & - & 21,0 & 22 & 57,3 & \\
\hline $19-9-921$ & 5,5 & 03 & 2,7 & 10 & 16,2 & \\
\hline $11-6-922\left({ }^{38}\right)$ & 11,7 & $02 \quad 15$ & 6,0 & 13 & 27,7 & \\
\hline $18-6-922$ & 12,2 & 0330 & 2,7 & - & 12,5 & \\
\hline $30-8-922$ & 20,0 & 0200 & 10,0 & 33 & 18,2 & \\
\hline $24-8-923\left({ }^{39}\right)$ & 10,5 & 0200 & 4,5 & 17 & 15,9 & \\
\hline $9-7-924$ & 12,5 & 0300 & 4,0 & 22 & 11,0 & \\
\hline $17-7-924$ & 17,6 & 0250 & 14,0 & 26 & 32,3 & \\
\hline $3-8-924$ & 9,6 & 0150 & 6,0 & 10 & 36,0 & \\
\hline $16-8-924$ & 18,5 & 0100 & 18,0 & 50 & 21,6 & \\
\hline
\end{tabular}




\begin{tabular}{|c|c|c|c|c|c|c|}
\hline Data & $\begin{array}{c}\text { Totale } \\
\text { della } \\
\text { precipit. } \\
\text { in } \\
\text { mm }\end{array}$ & $\begin{array}{c}\text { Durata } \\
\text { totale } \\
\text { della } \\
\text { precipit. } \\
\text { in } \\
\text { ore min. }\end{array}$ & $\begin{array}{c}\text { Massimo } \\
\text { della } \\
\text { precipit. } \\
\text { in } \\
\text { mm }\end{array}$ & $\begin{array}{c}\text { Durata } \\
\text { del } \\
\text { massimo } \\
\text { della } \\
\text { precipit. } \\
\text { in min. }\end{array}$ & $\begin{array}{c}\text { Intensità } \\
\text { oraria } \\
\text { del max } \\
\text { in } \\
\text { mn/h }\end{array}$ & $N o \operatorname{te}$ \\
\hline $27-3-925$ & - & - & 5,0 & 15 & 20,0 & (40) Anno piuttosto secco \\
\hline $16-5-925$ & 12,0 & 50 & 10,0 & 26 & 23,1 & durante i mesi estivi \\
\hline $7-5-925$ & 16,0 & 45 & 10,0 & 22 & 27,3 & (11) Fase più intensa \\
\hline $22-6-925$ & 10,2 & 0115 & 7,7 & 31 & 14,9 & (42) Mancan i nluxio \\
\hline $9-7-925$ & 17,5 & 0245 & 10,5 & 17 & 37,1 & ( ${ }^{(2)}$ ) Mancano i pluvio- \\
\hline $15-7-925$ & 16,8 & 0310 & 9,0 & 43 & 12,6 & grammi dal 11-2 al 3-6 \\
\hline $20-7-925$ & 19,1 & 56 & 18,0 & 17 & 63,5 & \\
\hline $24-8-925$ & 13,9 & 0140 & 6,5 & 23 & 17,0 & \\
\hline $25-4-926$ & 27,0 & 0615 & 17,8 & 56 & 19,1 & \\
\hline $3-5-926$ & 7,7 & 0130 & 6,0 & 17 & 21,2 & \\
\hline $7-5-926$ & 10,8 & 0110 & 6,7 & 26 & 15,5 & \\
\hline $8-7-926$ & & & 4,5 & 22 & 12,3 & \\
\hline $8-8-926$ & 9,4 & 45 & 9,1 & 26 & 21,0 & \\
\hline $28-10-926$ & 9,0 & 0500 & 5,3 & 4 & 79,5 & \\
\hline $2-11-926$ & 55,2 & 1245 & 18,5 & 60 & 18,5 & \\
\hline $29-4-928\left({ }^{40}\right)$ & 10,3 & 0210 & 4,5 & 4 & 67,5 & \\
\hline $23-8-928$ & 8,1 & 43 & 8,1 & 43 & 11,3 & \\
\hline $30-8-928$ & 10,2 & 0140 & 7,0 & 13 & 32,3 & \\
\hline 12-9-928 & 10,2 & 0220 & 7,5 & 17 & 26,5 & \\
\hline $16-9-928$ & 48,0 & 0120 & 33,2 & 29 & 68,7 & \\
\hline $16-9-928\left(^{41}\right)$ & & & 4,7 & 4 & 70,5 & \\
\hline $20-6-929\left(^{42}\right)$ & 28,5 & 0100 & 28,0 & 36 & 46,7 & \\
\hline $28-6-929$ & 11,0 & 0100 & 11,0 & 60 & 11,0 & \\
\hline 9-8-929 & 10,5 & 0230 & 4,5 & 9 & 30,0 & \\
\hline 9-8-929 & 6,5 & 26 & 5,0 & 10 & 30,0 & \\
\hline $10-8-929$ & 16,2 & 0700 & 5,5 & 7 & 47,1 & \\
\hline 11-9-929 & 13,0 & 54 & 10,0 & 26 & 23,1 & \\
\hline $19-10-929$ & - & - & 3,2 & 4 & 48,0 & \\
\hline $26-10-929$ & 22,0 & 07 & 7,0 & 26 & 16,2 & \\
\hline 29-4-930 & - & - & & 13 & 23,1 & \\
\hline $24-6-930$ & & - & 8,5 & 17 & 30,0 & \\
\hline $27-6-930$ & 11,2 & 43 & 10,0 & 22 & 27,3 & \\
\hline $2-7-930$ & 22,1 & 0105 & 18,0 & 36 & 30,0 & \\
\hline $10-7-930$ & 24.0 & 01 & 7,5 & 9 & 50,0 & \\
\hline $10-7-930$ & 24,0 & 01 & 7,5 & & 50,0 & \\
\hline $10-7-930$ & - & - & 9,0 & 20 & 27,0 & \\
\hline $24-7-930$ & 16,0 & 0240 & & 20 & & \\
\hline 5-8-930 & 8,0 & 40 & 5,0 & 10 & 30,0 & \\
\hline $22-10-930$ & 11,3 & 0130 & 9,0 & 22 & 24,5 & \\
\hline
\end{tabular}




\begin{tabular}{|c|c|c|c|c|c|c|}
\hline Data & $\begin{array}{c}\text { Totale } \\
\text { della } \\
\text { precipit. } \\
\text { in } \\
\text { mm }\end{array}$ & $\begin{array}{c}\text { Durata } \\
\text { totale } \\
\text { della } \\
\text { precipit. } \\
\text { in } \\
\text { ore min. }\end{array}$ & $\begin{array}{c}\text { Massimo } \\
\text { della } \\
\text { precipit. } \\
\text { in } \\
\text { mm }\end{array}$ & $\begin{array}{l}\text { Durata } \\
\text { del } \\
\text { massimo } \\
\text { della } \\
\text { precipit. } \\
\text { in min. }\end{array}$ & $\begin{array}{c}\text { Intensità } \\
\text { oraria } \\
\text { del max } \\
\text { in } \\
\mathrm{mm} / \mathrm{h}\end{array}$ & $\mathrm{Note}$ \\
\hline $30-4-931$ & 20,3 & 0240 & 12,5 & 36 & 20,8 & $\left.{ }^{(43}\right)$ Fase più intensa \\
\hline $3-5-931$ & 26,0 & 0100 & 26,0 & 60 & 26,0 & (44) Come unica \\
\hline $31-5-931$ & 8,2 & 0220 & 5,7 & 21 & 16,3 & (45) Come precipit. unica \\
\hline $20-7-931$ & 6,7 & 45 & 5,4 & 17 & 19,0 & \\
\hline $\begin{array}{r}20-7-931 \\
9-8-931\end{array}$ & $\begin{array}{r}7,4 \\
24,3\end{array}$ & $\begin{array}{ll}01 & 00 \\
04 & 13\end{array}$ & $\begin{array}{r}5,7 \\
18,4\end{array}$ & $\begin{array}{l}17 \\
21\end{array}$ & $\begin{array}{l}20,1 \\
52,6\end{array}$ & $\begin{array}{l}\left({ }^{(0)}\right) \text { I tase } \\
\left({ }^{47}\right) \text { ll fase }\end{array}$ \\
\hline $24-8-931$ & 60,5 & 1210 & 11,0 & 60 & 11,0 & \\
\hline $4-9-931$ & 23,0 & 0315 & 13,4 & 17 & 47,3 & \\
\hline $2-5-932$ & 11,2 & 01 & 11,0 & 21 & 31,4 & \\
\hline $22-5-932$ & 11,5 & 50 & 8,0 & 9 & 53,3 & \\
\hline $16-6-932$ & 19,1 & 45 & 18,5 & 42 & 26,4 & \\
\hline $8-7-932$ & 15,0 & $03 \quad 10$ & 10,0 & 19 & 31,6 & \\
\hline $3-8-932$ & 7,5 & 30 & 6,8 & 17 & 24,0 & \\
\hline 8-9-932 & 20,6 & 0135 & 16,0 & 23 & 41,7 & \\
\hline $30-9-932$ & 12,5 & 0200 & 6,0 & 17 & 21,2 & \\
\hline $17-4-933$ & 23,0 & 04 & 18,0 & 60 & 18,0 & \\
\hline $17-4-933\left({ }^{43}\right)$ & & & 10,0 & 26 & 21,8 & \\
\hline $1-6-933$ & 13,5 & 30 & 13,5 & 30 & 27,0 & \\
\hline $23,6,933$ & 10,8 & 01 & 10,2 & 30 & 20,4 & \\
\hline $22-7-933$ & 31,9 & 0430 & 24,2 & 43 & 33,8 & \\
\hline $30-7-933\left({ }^{44}\right)$ & 49,0 & $01 \quad 14$ & - & & 39,7 & \\
\hline $30-7-933$ & - & - & 35,4 & 36 & 59,0 & \\
\hline $30-7-933$ & - & 一 & 13,6 & 36 & 22,7 & \\
\hline $7-8-933$ & 14,5 & 0126 & 12,8 & 26 & 29,5 & \\
\hline $15-8-933$ & 20,8 & 01 & 18,0 & 17 & 63,5 & \\
\hline $23-10-933$ & 13,1 & 0350 & 8,7 & 22 & 23,7 & \\
\hline $22-3-934$ & 17,5 & 35 & 17,5 & 35 & 30,0 & \\
\hline $3-5-934$ & 34,3 & 0202 & 23,2 & 17 & 81,9 & \\
\hline $26-5-934$ & 13,1 & 13 & 13,1 & 13 & 60,5 & \\
\hline $3-6-934$ & - & - & 4,0 & 13 & 18,5 & \\
\hline $31-7-934$ & 16,6 & 17 & 16,6 & 17 & 58,6 & \\
\hline $14-8-934$ & 12,5 & 0140 & 7,5 & 17 & 26,5 & \\
\hline $22-8-934$ & 12,7 & 0530 & 10,0 & 4 & 150,0 & \\
\hline $1-9-934$ & 11,6 & 0320 & 8,5 & 26 & 19,6 & \\
\hline $13-5-935$ & 26,0 & 0148 & 23,0 & 28 & 49,3 & \\
\hline $16-5-935$ & - & - & 6,0 & 13 & 27,7 & \\
\hline $1-6-935$ & - & - & 4,5 & 13 & 20,8 & \\
\hline $3-7-935$ & 18,7 & 0150 & 10,0 & 22 & 27,3 & \\
\hline $14-8-935\left({ }^{45}\right)$ & 60,6 & 1045 & 44,0 & 83 & 31,8 & \\
\hline $14-8-935\left({ }^{46}\right)$ & - & - & 20,0 & 26 & 46,1 & \\
\hline $14-8-935\left({ }^{17}\right)$ & - & - & 12,0 & 26 & 27,7 & \\
\hline $14-8-935$ & - & - & 10,0 & 22 & 27,3 & \\
\hline $25-8-935$ & 26,2 & $02 \quad 10$ & 13.5 & 43 & 18,8 & \\
\hline $12-10-935$ & & & 11,7 & 17 & 41,3 & \\
\hline
\end{tabular}


PRECIPITAZIONI DI NOTEVoli INTENSITA A PAVIA DAL 1908 AL 1957

Seguito Tab. I (f)

G. PANNOCCIIIA

\begin{tabular}{|c|c|c|c|c|c|c|}
\hline Data & $\begin{array}{c}\text { Totale } \\
\text { della } \\
\text { precipit. } \\
\text { in } \\
\text { mm }\end{array}$ & $\begin{array}{c}\text { Durata } \\
\text { totale } \\
\text { della } \\
\text { precipit. } \\
\text { in } \\
\text { ore min. }\end{array}$ & $\begin{array}{l}\text { Massimo } \\
\text { della } \\
\text { precipit. } \\
\text { in } \\
\text { mm }\end{array}$ & $\begin{array}{c}\text { Durata } \\
\text { del } \\
\text { massimo } \\
\text { della } \\
\text { precipit. } \\
\text { in min. }\end{array}$ & $\begin{array}{c}\text { Intensità } \\
\text { oraria } \\
\text { del max } \\
\text { in } \\
\mathrm{mm} / \mathrm{h}\end{array}$ & Note \\
\hline $22-5-936$ & 14,7 & 0140 & 10,0 & 16 & 23,1 & (48) Fase più intensa. \\
\hline $24-5-936$ & $\mathbf{5}, \mathbf{5}$ & 0100 & 5,0 & 24 & 12,5 & (49) Violento temp.; la \\
\hline $2-6-936$ & 17,5 & 0130 & 15,5 & 22 & 42,3 & fase più intensa è de. \\
\hline $4-6-936$ & 7,8 & 01 & 6,0 & 17 & 21,2 & finita uragano. \\
\hline $24-6-936$ & 13,1 & 0340 & 6,5 & 10 & 39,0 & \\
\hline $26-6-936$ & 6,2 & 0130 & 2,8 & 3 & 56,0 & \\
\hline $2-7-936$ & 7,3 & - & 4,5 & 9 & 26,7 & \\
\hline $30-7-936$ & 11,6 & 50 & 10,0 & 24 & 25,0 & \\
\hline $13-4-937$ & 78,3 & 26 & 27,5 & 60 & 27,5 & \\
\hline $9-5-937$ & - & - & 6,5 & 24 & 16,2 & \\
\hline $18,6-937$ & - & - & 10,0 & 5 & 120,0 & \\
\hline $18-6-937$ & - & - & 12,0 & 35 & 20,6 & \\
\hline $31-8-937$ & - & - & 3,0 & 13 & 13,8 & \\
\hline $12-8-937$ & 25,8 & 3 & 23,0 & 23 & 60,0 & \\
\hline $10-8-937$ & - & - & 5.7 & 19 & 18,0 & \\
\hline $20-9-937$ & 8,9 & - & 8,0 & 23 & 20,8 & \\
\hline $29-11-937$ & 6,3 & 0150 & 5,8 & 19 & 18,3 & \\
\hline $17-5-938\left(^{(48}\right)$ & 52.7 & $08 \quad 30$ & 19.2 & 22 & 52,3 & \\
\hline $17-5-938$ & & - & 39,2 & 87 & 27,0 & \\
\hline $13-6-938$ & 29,8 & 0120 & 16,0 & 22 & 43,6 & \\
\hline $30-6-938$ & 26,0 & 0105 & 20,0 & 19 & 63,2 & \\
\hline $15-7-938$ & 9,7 & 0243 & 5,7 & 17 & 20,0 & \\
\hline $24-7-938$ & 12,2 & 40 & 11,2 & 26 & 25,8 & \\
\hline $21-8-938$ & 19,6 & 0300 & 13,0 & 26 & 30,0 & \\
\hline $28-8-938$ & 121,1 & 1230 & 20,0 & 22 & 54,4 & \\
\hline $28-8-938$ & - & - & 20,0 & 31 & 38,7 & \\
\hline $28-8-938$ & - & - & 17,5 & 17 & 61,8 & \\
\hline $28-8-938$ & - & - & 20,0 & 30 & 40,0 & \\
\hline $12-5-939$ & 18,4 & 0300 & 10,0 & 17 & 35,3 & \\
\hline $31-5-939$ & 12,5 & 0145 & 5,0 & 10 & 30,0 & \\
\hline $12-6-939$ & 10,0 & 0120 & 5,0 & 8 & 37,5 & \\
\hline $12-6-939$ & 12,8 & 0305 & 7,4 & 9 & 49,3 & \\
\hline $5-8-939$ & 10,2 & 0100 & 5,2 & 10 & 31,2 & \\
\hline $20-8-939$ & 11,7 & $01 \quad 15$ & 10,0 & 18 & 33,3 & \\
\hline $23-8-939$ & 7,0 & 0200 & 6,2 & 9 & 41,3 & \\
\hline $12-9-939\left({ }^{4 \theta}\right)$ & 46,4 & 0350 & 7,2 & 13 & 33,2 & \\
\hline $12-9-939$ & -1 & - & 36,5 & 56 & 39,1 & \\
\hline $12-9-939$ & - & - & 4,5 & 10 & 27,1 & \\
\hline $12-9-939$ & - & - & 10,0 & 15 & 40,0 & \\
\hline $12-9-939$ & - & - & 11,0 & 5 & 132,0 & \\
\hline $5-10-939$ & 18,2 & $02 \quad 10$ & 4,7 & 7 & 40,2 & \\
\hline $7-10-939$ & 47,1 & 1000 & 8,0 & 17 & 28,2 & \\
\hline
\end{tabular}


PRECIPITAZIONI DI NOTEVoJ. INTENSITA A PAVIA DAL 1908 AL 1957

G. PANNOCCHIA

Seguito Tab. I (g)

\begin{tabular}{|c|c|c|c|c|c|c|}
\hline Data & $\begin{array}{c}\text { Totale } \\
\text { della } \\
\text { precipit. } \\
\text { in } \\
\text { mm }\end{array}$ & $\begin{array}{c}\text { Durata } \\
\text { totale } \\
\text { della } \\
\text { precipit. } \\
\text { in } \\
\text { ore min. }\end{array}$ & $\begin{array}{c}\text { Massimo } \\
\text { della } \\
\text { precipit. } \\
\text { in } \\
\text { min }\end{array}$ & $\begin{array}{l}\text { Durata } \\
\text { del } \\
\text { massimo } \\
\text { della } \\
\text { precipit. } \\
\text { in min. }\end{array}$ & $\begin{array}{c}\text { Intensità } \\
\text { oraria } \\
\text { del max } \\
\text { in } \\
\text { mm } / h\end{array}$ & Not $\theta$ \\
\hline $30-4-940$ & - & - & 9,0 & 30 & 18,0 & (50) Fase meno intensa. \\
\hline $3-5-940$ & - & - & 6,0 & 18 & 20,0 & (51) Fase meno intensa. \\
\hline $17-5-940$ & 32,9 & 1540 & 5,8 & 26 & 13,5 & (52) Fase più intensa. \\
\hline $29-5-940$ & 49,2 & 1205 & 6,0 & 18 & 20,0 & ${ }^{\left({ }^{2}\right)}$ Fase più intensa. \\
\hline $29-5-940\left({ }^{50}\right)$ & - & - & 10,0 & 44 & 13,6 & (53) Anno notevolmente \\
\hline $29-5-940\left(^{51}\right)$ & - & - & 4,0 & 13 & 18,5 & secco. \\
\hline $16-6-940$ & 37,2 & 07 & 7,0 & 26 & 16,2 & \\
\hline $16-6-940\left({ }^{52}\right)$ & - & - & 13,5 & 43 & 18,8 & \\
\hline $11-6-940$ & 13,8 & 0330 & 10,0 & 42 & 14,3 & \\
\hline 4--7-940 & 63,0 & $0 ! 40$ & 63,0 & 100 & 38,0 & \\
\hline $8-7-940$ & - & - & 8,0 & 26 & 18,5 & \\
\hline $12,8,940$ & 41,4 & 0145 & 39,7 & 35 & 68,0 & \\
\hline $9-9-940$ & 14,8 & $02 \quad 15$ & 9,5 & 26 & 21,9 & \\
\hline $1-10-940$ & - & - & 15,8 & 18 & 52,7 & \\
\hline $26-10-940$ & - & - & 4,0 & 09 & 26,7 & \\
\hline $4,3-941$ & - & - & 6,5 & 26 & 15,0 & \\
\hline 5-6-941 & 17,9 & 36 & 17,9 & 36 & 29,8 & \\
\hline $12-7-941$ & - & - & 7,7 & 35 & 13,2 & \\
\hline $16-7-941$ & - & - & 10,6 & 30 & 21,2 & \\
\hline $27-7-941$ & 35,8 & 0110 & 14,6 & 60 & 14,6 & \\
\hline 28-7-941 & 22,1 & 0215 & 31,0 & 60 & 31,0 & \\
\hline 8-9-941 & 9,6 & 0140 & 6,6 & 22 & 18,0 & \\
\hline $29-9-941$ & 16,0 & 0200 & 4,5 & 17 & 15,9 & \\
\hline $30-9-941$ & 57,0 & 1100 & $12-5$ & 30 & 25,0 & \\
\hline $23-10-941$ & 14,3 & 0445 & 9,0 & 21 & 25,7 & \\
\hline $2-7-942\left(^{53}\right)$ & 12,9 & 45 & 10,8 & 17 & 38,1 & \\
\hline $19-7-942$ & 6,6 & 35 & 4,0 & 12 & 20,0 & \\
\hline $25-9-942$ & 30,0 & 0150 & 28,5 & 24 & 71,2 & \\
\hline $2-6-943$ & 17,2 & 0100 & 14,0 & 13 & 64,6 & \\
\hline $2-6-943$ & 41,4 & 0250 & 30,0 & 25 & 72,0 & \\
\hline $30-6-943$ & 19,8 & 0135 & 14,0 & 24 & 35,0 & \\
\hline $28-8-943$ & 10,7 & 0340 & 7,0 & 30 & 14,0 & \\
\hline $4-9-943$ & 23,0 & 0220 & 11,0 & 17 & 38,0 & \\
\hline $17-4-944$ & 13,1 & 0300 & 7,0 & 14 & 30,0 & \\
\hline $17-6-944$ & 21,5 & $01 \quad 12$ & 19,0 & 17 & 67,0 & \\
\hline $9-7-944$ & 17,2 & 30 & 10,8 & 25 & 25,9 & \\
\hline $21-7-944$ & 26,1 & 50 & 26,0 & 25 & 62,4 & \\
\hline $10-8-944$ & 6,2 & 50 & 5,5 & 14 & 23,5 & \\
\hline 3-9-944 & - & - & 6,5 & 13 & 30,0 & \\
\hline $6-10-944$ & - & - & 9,7 & 18 & 32,3 & \\
\hline 2-11-944 & - & - & 3,7 & 10 & 22,2 & \\
\hline
\end{tabular}


PRECIPITAZIONI DI NOTEVoli INTENSITÀ A PAVIA DAL 1908 AL 1957

Seguito Tab. I (h)

G. PAN NOCCHIA

\begin{tabular}{|c|c|c|c|c|c|c|}
\hline Data & $\begin{array}{c}\text { Totale } \\
\text { della } \\
\text { precipit. } \\
\text { in } \\
\text { mm }\end{array}$ & $\begin{array}{c}\text { Durata } \\
\text { totale } \\
\text { della } \\
\text { precipit. } \\
\text { in } \\
\text { ore min. }\end{array}$ & $\begin{array}{c}\text { Massimo } \\
\text { della } \\
\text { precipit. } \\
\text { in } \\
\mathrm{mm}\end{array}$ & $\begin{array}{c}\text { Durata } \\
\text { del } \\
\text { massimo } \\
\text { della } \\
\text { precipit. } \\
\text { in min. }\end{array}$ & $\begin{array}{c}\text { Intensità } \\
\text { oraria } \\
\text { del max } \\
\text { in } \\
\mathrm{mm} / \mathrm{h}\end{array}$ & Note \\
\hline $26-5-945$ & 20,5 & $03 \quad 17$ & 10,5 & 17 & 37,0 & - \\
\hline $13-6-945$ & & - & 3,3 & 9 & 22,0 & \\
\hline $7-8-945$ & 19,3 & $01 \quad 15$ & 11,0 & 19 & 34,7 & \\
\hline $9-8-945$ & 18,6 & 30 & 18,4 & 24 & 46,0 & \\
\hline $8-8-945$ & - & - & 4,0 & 17 & 14,1 & \\
\hline $8-8-945$ & - & - & 5,5 & 18 & 18,3 & \\
\hline $8-8-945$ & - & - & 5,9 & 30 & 11,8 & \\
\hline $9-8-945$ & 18,7 & 0100 & 16,2 & 16 & 60,7 & \\
\hline $13-8-945$ & 30,2 & 0110 & 26,5 & 17 & 93,5 & \\
\hline $6-5-946$ & 一 & - & 4,7 & 17 & 16,6 & \\
\hline $23-6-946$ & 28,0 & 0125 & 25,5 & 77 & 19,9 & \\
\hline $6-7-946$ & 25,2 & 0500 & 13,5 & 21 & 38,6 & \\
\hline $7-7-946$ & 19,7 & 0310 & 11,0 & 60 & 11,0 & \\
\hline $15-7-946$ & 13,0 & 0300 & 9,8 & 26 & 22,6 & \\
\hline $21-7-946$ & 5,4 & 50 & 5,2 & 11 & 28,0 & \\
\hline $21-7-946$ & 25,0 & 0110 & 24,7 & 13 & 114,0 & \\
\hline $19-8-946$ & 7,7 & $01 \quad 15$ & 7,0 & 26 & 16,7 & \\
\hline $22-8-946$ & 14,7 & 0325 & 9,0 & 28 & 19,3 & \\
\hline $26-8-946$ & 17,8 & 50 & 17,5 & 25 & 42,0 & \\
\hline $31-8-946$ & - & - & 6,0 & 13 & 27,7 & \\
\hline $31-8-946$ & 17,6 & 0220 & 7,2 & 28 & 15,4 & \\
\hline $6-9-946$ & - & - & 3,5 & 12 & 17,5 & \\
\hline $22-10-946$ & - & - & 6,2 & 23 & 13,3 & \\
\hline $9-10-947$ & 21,1 & 0230 & 17,0 & 60 & 17,0 & \\
\hline $10-5-947$ & 8,9 & $01-$ & 4,5 & 13 & 20,8 & \\
\hline $20-5-947$ & 17,9 & 55 & 16,9 & 33 & 30,7 & \\
\hline $14-6-947$ & 25,5 & 0426 & 19,3 & 51 & 19,3 & \\
\hline $19-6-947$ & 7,8 & 0210 & 6,0 & 30 & 12,0 & \\
\hline $25-9-947$ & - & क & 21,5 & 43 & 30,0 & \\
\hline $25-4-948$ & 14,6 & 0150 & 13,6 & 50 & 16,3 & \\
\hline $21-7-948$ & 18,9 & & 18,0 & 18 & 60,0 & \\
\hline $39-5-948$ & - & - & 5,8 & 14 & 24,9 & \\
\hline $5-7-948$ & - & - & 5,6 & 21 & 16,0 & \\
\hline $5-8-948$ & 8,3 & $01 \quad 15$ & 7,8 & 35 & 13,4 & \\
\hline $10-8-948$ & 8,8 & 0310 & 6,0 & 30 & 12,0 & \\
\hline 4-9-948 & 84,5 & 0520 & 39,0 & 48 & 48,7 & \\
\hline 4-9-948 & - & - & 22,0 & 25 & 50,8 & \\
\hline $12-9-948$ & 139,1 & 10 & 61,7 & 59 & 62,7 & \\
\hline $12-9-948$ & - & - & 22,4 & 55 & 24,4 & \\
\hline $17-7-949$ & 100,0 & 04 & 76,2 & 60 & 76,2 & \\
\hline $18-7-949$ & 21,6 & $04 \quad 10$ & 8,5 & 27 & 18,9 & \\
\hline $24-7-949$ & 5,0 & 0120 & 3,8 & 21 & 10,9 & \\
\hline 8-9-949 & 22,0 & 0130 & 15,0 & 26 & 34,6 & \\
\hline $16-9-949$ & 31,5 & 0210 & 30,0 & 45 & 40,0 & \\
\hline
\end{tabular}




\begin{tabular}{|c|c|c|c|c|c|c|}
\hline Data & $\begin{array}{c}\text { Totale } \\
\text { della } \\
\text { precipit. } \\
\text { in } \\
\text { mm }\end{array}$ & $\begin{array}{c}\text { Durata } \\
\text { totale } \\
\text { della } \\
\text { procipit. } \\
\text { in } \\
\text { ore min. }\end{array}$ & $\begin{array}{l}\text { Massimo } \\
\text { della } \\
\text { precipit. } \\
\text { in } \\
\text { mm }\end{array}$ & $\begin{array}{c}\text { Durata } \\
\text { del } \\
\text { massimo } \\
\text { della } \\
\text { precipit. } \\
\text { in min. }\end{array}$ & $\begin{array}{c}\text { Intensità } \\
\text { oraria } \\
\text { del max } \\
\text { in } \\
\mathrm{mm} / \mathrm{h}\end{array}$ & Note \\
\hline $9-5-950$ & 9.4 & 0125 & & & & \\
\hline $17-6-950$ & $\begin{array}{r}9,4 \\
12,6\end{array}$ & $\begin{array}{l}0125 \\
0150\end{array}$ & $\begin{array}{l}8,0 \\
7,5\end{array}$ & $\begin{array}{l}26 \\
17\end{array}$ & $\begin{array}{l}18,5 \\
26,5\end{array}$ & \\
\hline $25-6-950$ & 18,2 & 05 & 5,5 & 26 & 12,7 & \\
\hline $28-7-950$ & 27,2 & 0130 & 26,2 & 24 & 65,5 & \\
\hline $17-8-950$ & 15,3 & 0215 & 10,0 & 26 & 23,1 & \\
\hline $21-9-950$ & 11,5 & 0105 & 8,0 & 29 & 16,6 & \\
\hline $23-6-951$ & 13,6 & 0125 & 7,0 & 36 & 11,7 & \\
\hline $31-8-951$ & 4,0 & 20 & 4,0 & 20 & 12,0 & \\
\hline $22-10-951$ & - & - & 11,2 & 47 & 14,3 & \\
\hline $11-11-951$ & - & - & 4,5 & 17 & 15,0 & \\
\hline $11-11-951$ & - & - & 12,0 & 30 & 24,0 & \\
\hline $8-5-952$ & - & - & 7,0 & 36 & 11,7 & \\
\hline $30-5-952$ & 52,2 & 0230 & 51,5 & 41 & 75,5 & \\
\hline $9-6-952$ & 18,8 & $03-$ & 12,0 & 60 & 12,0 & \\
\hline $8-7-952$ & 6,0 & 10 & 6,0 & 10 & 36,0 & \\
\hline $9-7-952$ & 34,5 & 0430 & 25,2 & 34 & 44,5 & \\
\hline $17-7-952$ & 21,9 & 0110 & 21,0 & 51 & 24,7 & \\
\hline $20-8-952$ & 5,5 & 50 & 4,7 & 26 & 10,8 & \\
\hline $24-8-952$ & - & - & 11,0 & 34 & 19,4 & \\
\hline $21-10-952$ & - & - & 8,0 & 43 & 11,2 & \\
\hline $8-6-953$ & 29,2 & 0315 & 20,0 & 45 & 26,7 & \\
\hline $24-6-953$ & 10,2 & 0230 & 10,0 & 41 & 14,6 & \\
\hline $30-7-953$ & 17,3 & 0145 & 15,0 & 30 & 30,0 & \\
\hline $3-8-953$ & 35,8 & 0540 & 23,0 & 60 & 23,0 & \\
\hline $3-8-953$ & 5,0 & 30 & 4,8 & 18 & 16,0 & \\
\hline $9-8-953$ & & - & 5,0 & 09 & 33,3 & \\
\hline $26-8-953$ & 33,3 & $11-$ & 17,5 & 38 & 27,6 & \\
\hline $25-9-953$ & & - & 10,0 & 17 & 35,3 & \\
\hline $20-10-953$ & 7,1 & 0130 & 4,0 & 09 & 26,7 & \\
\hline $27-10-953$ & 43,0 & 08 & 22,5 & 60 & 22,5 & \\
\hline $3-5-954$ & 9,4 & 0105 & 9,0 & 34 & 15,9 & \\
\hline $12-5-954$ & & & 4,5 & 13 & 20,8 & \\
\hline $23-5-954$ & 5,1 & 40 & 4,5 & 21 & 12,9 & \\
\hline $2-6-954$ & 10,5 & & 9,5 & 14 & 40,7 & \\
\hline $6-6-954$ & 23,7 & 0320 & 12,0 & 60 & 12,0 & \\
\hline $15-6-954$ & - & - & 12,0 & 26 & 27,7 & \\
\hline $21-6-954$ & 5,4 & 30 & 5,3 & 22 & 14,5 & \\
\hline $23-6-954$ & 9,0 & 0245 & 6,5 & 21 & 18,6 & \\
\hline $30-6-954$ & 12,0 & $01 \quad 15$ & 9,5 & 15 & 38,0 & \\
\hline $7-7-954$ & 10,6 & $02-$ & 8,2 & 26 & 18,9 & \\
\hline $10-8-954$ & & - & 22,0 & 43 & 30,7 & \\
\hline $15-8-954$ & 15,2 & 1240 & 10,0 & 39 & 15,4 & \\
\hline
\end{tabular}




\begin{tabular}{|c|c|c|c|c|c|c|}
\hline Data & $\begin{array}{c}\text { Totale } \\
\text { della } \\
\text { precipit. } \\
\text { in } \\
\text { mm }\end{array}$ & $\begin{array}{c}\text { Durata } \\
\text { totale } \\
\text { della } \\
\text { precipit. } \\
\text { in } \\
\text { ore min. }\end{array}$ & $\begin{array}{c}\text { Massimo } \\
\text { della } \\
\text { precipit. } \\
\text { in } \\
\text { mm }\end{array}$ & $\begin{array}{c}\text { Durata } \\
\text { del } \\
\text { massimo } \\
\text { della } \\
\text { procipit. } \\
\text { in min. }\end{array}$ & $\begin{array}{c}\text { Intensità } \\
\text { oraria } \\
\text { del max } \\
\text { in } \\
\mathrm{mm} / \mathbf{l}\end{array}$ & Note \\
\hline $22-8-954$ & 37,3 & 0230 & 33,7 & 56 & 36,1 & \\
\hline $25-8-954$ & - & - & 15,0 & 35 & 25,7 & \\
\hline $8-9-954$ & - & - & 15,0 & 35 & 25,7 & \\
\hline $28-12-954$ & 一 & 一 & 6,0 & 24 & 15,0 & \\
\hline $11-5-955$ & 17,5 & 0120 & 15,0 & 28 & 32,1 & \\
\hline $21-5-955$ & & - & 4,5 & 17 & 15,9 & \\
\hline $11-6-955$ & 14,6 & 0255 & 7,0 & 36 & 11,7 & \\
\hline $19-6-955$ & - & - & 8,5 & 50 & 10,2 & \\
\hline $20-6-955$ & 8,4 & 25 & 8,0 & 18 & 26,7 & \\
\hline $11-7-955$ & - & - & 2,7 & 08 & 20,2 & \\
\hline $25-7-955$ & 13,6 & 0105 & 10,0 & 10 & 60,0 & \\
\hline $26-7-955$ & 30,7 & 0420 & 16,0 & 60 & 16,0 & \\
\hline $27-7-955$ & 46,0 & 0340 & 24,0 & 45 & 32,0 & \\
\hline $15-8-955$ & 32,5 & 45 & 32,0 & 30 & 64,0 & \\
\hline $31-8-955$ & - & - & 7,2 & 30 & 14,4 & \\
\hline 14-9-955 & - & - & 2,7 & 13 & 12,5 & \\
\hline $24-5-956$ & - & - & 6,0 & 34 & 10,6 & \\
\hline $25-5-956$ & - & - & 4,0 & 09 & 26,7 & \\
\hline $17-6-956$ & - & - & 15,5 & 40 & 23,3 & \\
\hline $28-6-956$ & 2,0 & 05 & 2,0 & 05 & 24,0 & \\
\hline $2-7-956$ & 25,8 & 0125 & 23,0 & 26 & $\mathbf{5 3}, 0$ & \\
\hline $11-7-956$ & - & - & 4,5 & 20 & 13,5 & \\
\hline $1-8-956$ & 4,1 & & 3,0 & 17 & 10,6 & \\
\hline $22-8-956$ & 13,8 & 0140 & 8,5 & 40 & 12,8 & \\
\hline $5-10-956$ & 26,8 & 0145 & 23,8 & 34 & 42,0 & \\
\hline $23-3-957$ & - & - & 5,5 & 26 & 12,7 & \\
\hline $24-3-957$ & - & - & 4,6 & 15 & 16,0 & \\
\hline $10-7-957$ & 39,8 & 0220 & 30,0 & 30 & 60,0 & \\
\hline $14-7-957$ & 8,6 & $01-$ & 8,0 & 27 & 17,8 & \\
\hline $15-7-957$ & 8,2 & 0125 & 7,0 & 34 & 12,3 & \\
\hline $15-7-957$ & 9,8 & 50 & 8,5 & 30 & 17,0 & \\
\hline $15-7-957$ & 20,8 & 0130 & 16,5 & 26 & 38,1 & \\
\hline $18-7-957$ & 10,2 & 30 & 10,2 & 30 & 20,4 & \\
\hline $26-9-957$ & 8,9 & 0105 & 6,0 & 13 & 27,7 & \\
\hline
\end{tabular}




\begin{tabular}{|c|c|c|c|c|c|c|c|c|c|c|}
\hline Data & 1920 & 1913 & 1915 & 1919 & 1928 & 1934 & 1937 & 1944 & $\begin{array}{l}\text { Somme } \\
\text { medie }\end{array}$ & $\begin{array}{c}\text { Calcolo } \\
\text { giorno } \\
\text { medio }\end{array}$ \\
\hline 1 & - & - & - & - & - & - & - & - & - & - \\
\hline 2 & - & - & - & - & - & - & - & - & - & - \\
\hline 3 & - & - & - & - & - & - & - & - & - & - \\
\hline 4 & - & - & - & - & - & - & - & - & - & - \\
\hline 5 & - & 35,1 & - & - & - & - & - & - & 35,1 & $5 \times 1=5$ \\
\hline 6 & - & - & - & - & - & - & - & - & - & - \\
\hline 7 & 一 & - & - & - & - & - & - & - & - & - \\
\hline 8 & - & - & - & - & - & - & - & - & - & - \\
\hline 9 & - & - & - & - & - & - & - & - & - & - \\
\hline 10 & - & - & - & - & - & - & - & - & - & - \\
\hline 11 & - & - & - & - & - & - & - & - & - & - \\
\hline 12 & - & - & - & - & - & - & - & - & - & - \\
\hline 13 & - & - & - & - & - & - & 27.5 & - & 27,5 & $13 \times 1=13$ \\
\hline 14 & - & - & - & - & - & - & - & - & - & - \\
\hline 15 & - & - & - & - & - & - & - & - & - & - \\
\hline 16 & - & - & - & - & - & - & - & - & - & - \\
\hline 17 & - & - & - & - & - & - & - & 30.0 & 30,0 & $17 \times 1=17$ \\
\hline 18 & - & - & -- & - & - & - & - & - & - & - \\
\hline 19 & - & - & - & - & - & - & - & - & - & - \\
\hline 20 & - & - & - & - & - & - & - & - & - & - \\
\hline 21 & - & - & 51,2 & 94,0 & - & - & - & - & 145,2 & $21 \times 2=42$ \\
\hline 22 & - & - & - & - & - & 30.0 & - & - & 30,0 & $22 \times 1=22$ \\
\hline 23 & - & - & 50,4 & - & - & - & - & - & 50,4 & $23 \times 1=23$ \\
\hline 24 & - & - & - & - & - & - & - & - & - & - \\
\hline 25 & - & - & - & - & - & - & - & - & - & - \\
\hline 26 & - & - & - & - & - & - & - & - & - & - \\
\hline 27 & - & 30,9 & 一 & - & - & 一 & - & 一 & 30,9 & $27 \times 1=27$ \\
\hline 28 & - & - & - & - & - & - & - & - & - & - \\
\hline 29 & - & - & - & - & 67,5 & - & - & - & 67,5 & $29 \times 1=29$ \\
\hline 30 & - & - & - & - & - & - & - & - & - & - \\
\hline 31 & 31,9 & - & - & - & - & - & - & - & - & - \\
\hline Somme & 31,9 & 66,0 & 101,6 & 94,0 & 67,5 & 30,0 & 27,5 & 30,0 & 416,6 & 178 \\
\hline Medie & 31,9 & - & - & - & - & - & - & - & 46,3 & 19,9 \\
\hline Medio & - & 33,0 & 50,8 & 94,0 & 67,5 & 30,0 & 27,5 & 30,0 & - & \\
\hline Freq. & 1 & 2 & 2 & 1 & 1 & 1 & 1 & 1 & 9 & Anni 7 \\
\hline
\end{tabular}


$\begin{array}{lllllllllllllllllllll}\text { Data } & 1911 & 1913 & 1914 & 1915 & 1916 & 1925 & 1931 & 1932 & 1934 & 1935 & 1938 & 1939 & 1945 & 1947 & 1948 & 1952 & 1955 & 1956 & \text { Somme } & \text { Caicoio } \\ \text { giorno }\end{array}$ medie medio

\begin{tabular}{|c|c|c|c|c|c|c|c|c|c|c|c|c|c|c|c|c|c|c|c|c|}
\hline 1 & 一 & - & - & 一 & 一 & 一 & 一 & 一 & 一 & - & - & - & 一 & 一 & - & 一 & - & - & - & - \\
\hline 2 & 一 & - & 一 & - & 一 & 一 & - & 31,4 & - & - & - & - & - & - & - & - & - & - & 31.4 & $2 \times 1=2$ \\
\hline 3 & 一 & - & 一 & 一 & - & - & 26,0 & - & 81,9 & - & - & - & - & - & - & - & - & - & 107,9 & $3 \times 2=6$ \\
\hline 4 & 一 & - & - & 一 & - & 一 & - & - & - & - & 一 & - & - & - & - & - & - & - & - & - \\
\hline 5 & - & 一 & - & - & - & - & - & - & - & - & - & - & - & - & 一 & - & - & - & - & - \\
\hline 6 & 一 & - & - & - & 32,3 & - & - & - & - & - & - & - & - & 一 & - & - & - & - & 32,3 & $6 \times 1=6$ \\
\hline 7 & 一 & - & - & - & - & 27,3 & - & - & - & 一 & 一 & - & - & 一 & - & - & - & - & 27,3 & $7 \times 1=7$ \\
\hline 8 & — & 一 & 一 & - & 一 & - & - & - & - & - & - & - & - & 一 & - & - & - & - & - & - \\
\hline 9 & - & - & - & 一 & 一 & - & - & - & - & - & - & - & - & - & - & - & - & - & - & - \\
\hline 10 & - & - & - & - & - & - & - & - & - & - & - & - & - & - & - & - & - & - & - & - \\
\hline 11 & 一 & - & - & - & - & - & - & $=$ & - & - & - & - & - & - & - & - & 32,1 & - & 32,1 & $11 \times 1=11$ \\
\hline 12 & 一 & - & - & 一 & - & - & - & - & - & - & - & 35,3 & - & 一 & - & - & - & - & 35,3 & $12 \times 1=12$ \\
\hline 13 & - & 一 & 一 & - & - & - & - & - & - & 49,3 & - & - & - & - & - & - & - & - & 49,3 & $3 \times 1=13$ \\
\hline 14 & - & - & - & - & - & - & - & - & - & - & - & - & - & - & - & - & - & - & - & - \\
\hline 15 & 一 & $\overline{332}$ & - & - & - & - & - & - & - & - & - & - & - & - & - & - & - & - & - & - \\
\hline 16 & $\ldots$ & $\left\{\begin{array}{l}36,4 \\
42,0\end{array}\right.$ & - & - & - & - & - & - & - & 27,7 & - & - & - & - & - & - & - & - & 139,3 & $16 \times 4=64$ \\
\hline 17 & - & - & - & - & - & - & - & - & - & - & $\begin{array}{r}52.3 \\
\quad 26,0\end{array}$ & - & - & 一 & - & - & - & - & 79,3 & $17 \times 2=34$ \\
\hline 18 & - & - & 一 & - & - & - & - & - & - & 一 & - & - & - & - & - & 一 & - & - & - & - \\
\hline 19 & 一 & - & - & - & 一 & - & 一 & - & - & - & - & - & 一 & 一 & - & 一. & - & - & - & - \\
\hline 20 & 一 & - & - & - & - & - & - & - & - & - & - & - & - & 30,7 & - & - & - & - & 30,7 & $20 \times 1=20$ \\
\hline 21 & - & - & - & - & - & - & - & - & 一 & 一 & - & - & - & - & 60,0 & 一 & 一 & - & 60,0 & $21 \times 1=21$ \\
\hline 22 & 一 & - & - & - & - & - & - & 53,3 & - & - & - & - & - & 一 & - & - & - & - & 53,3 & $22 \times 1=22$ \\
\hline 23 & 一 & - & 一 & - & - & - & - & - & - & - & - & - & - & - & - & 一 & - & - & - & - \\
\hline 24 & - & - & 71,6 & - & - & - & - & - & - & - & - & - & - & - & - & - & - & - & 71,6 & $24 \times 1=24$ \\
\hline 25 & - & - & - & - & - & - & - & - & - & - & - & - & - & - & - & - & - & 26.7 & 26,7 & $25 \times 1=25$ \\
\hline 26 & - & - & - & - & - & - & - & - & 60,5 & -- & - & - & 37,0 & 一 & - & - & - & - & 97,5 & $26 \times 2=52$ \\
\hline 27 & - & - & - & 一 & 一 & - & - & - & - & 一 & - & - & - & - & - & - & - & - & - & - \\
\hline 28 & $\left\{\begin{array}{l}35,9 \\
73,5\end{array}\right.$ & - & - & - & - & - & - & - & - & - & - & - & - & 一 & 一 & - & - & - & 109,4 & $28 \times 2=56$ \\
\hline 29 & - & - & - & - & - & - & - & - & - & - & - & - & - & - & - & - & - & - & - & - \\
\hline 30 & - & - & - & - & - & - & - & - & - & - & - & - & - & - & - & 75,5 & - & - & 75,5 & $30 \times 1=30$ \\
\hline 31 & - & - & - & 72,0 & - & - & - & - & - & - & - & 30,0 & - & - & - & - & - & - & 102,0 & $31 \times 2=62$ \\
\hline
\end{tabular}

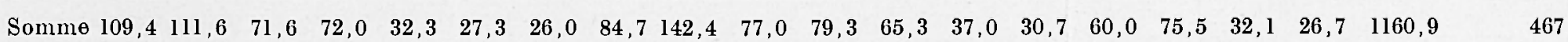

Medie - - - - - - - - - - - - - - - - - -

17,5

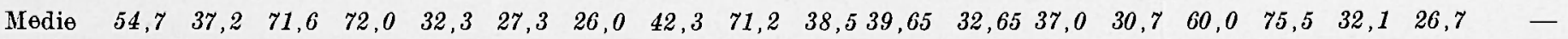

$\begin{array}{llllllllllllllllllllll}\text { Freq. } & 2 & 3 & 1 & 1 & 1 & 1 & 1 & 2 & 2 & 2 & 2 & 2 & 1 & 1 & 1 & 1 & 1 & 1 & 26 & \text { Anni } & 18\end{array}$

Mese M a g g i o 


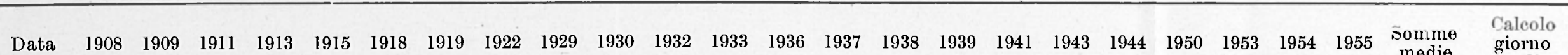
medie giorno

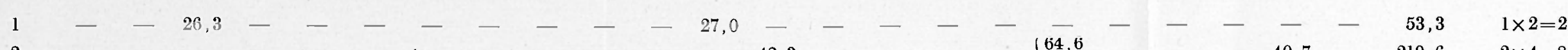

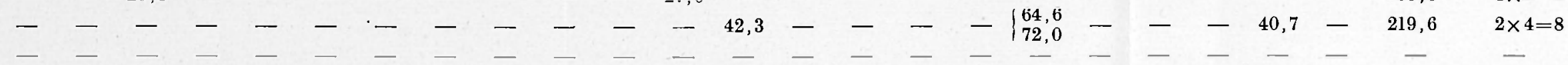

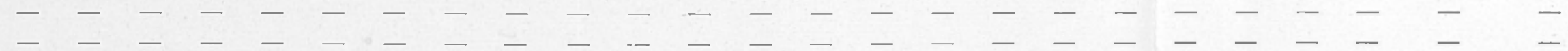

$$
\begin{aligned}
& -\quad-\quad-\quad-\left\{\begin{array}{r}
43,0 \\
162,0
\end{array}\right.
\end{aligned}
$$

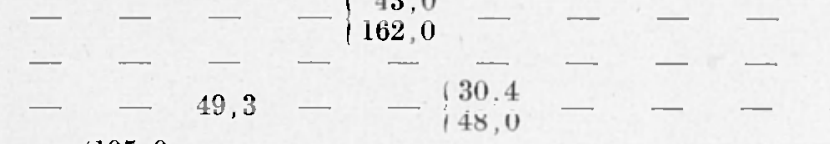

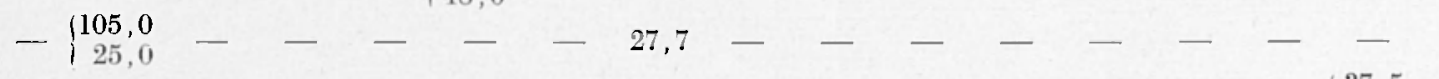

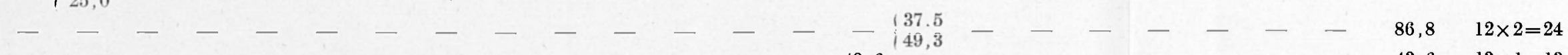

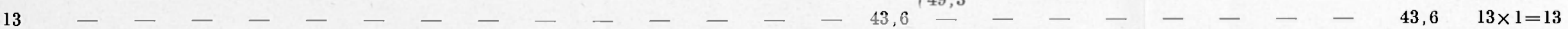

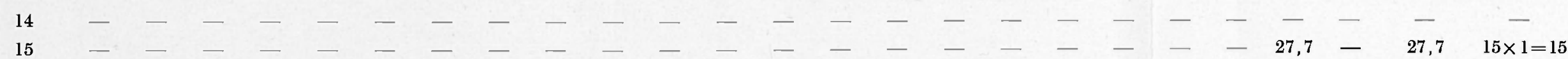

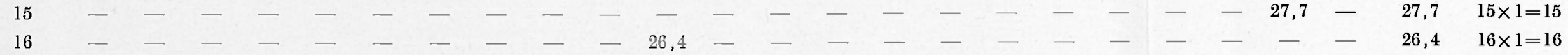

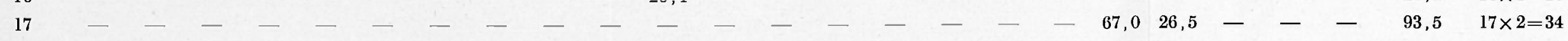

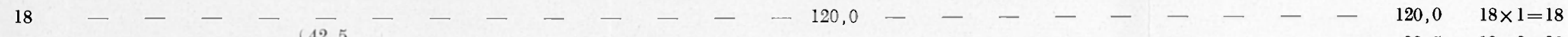

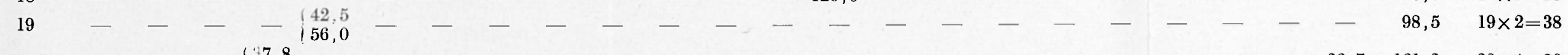

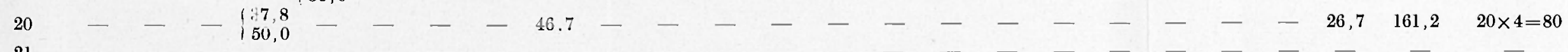

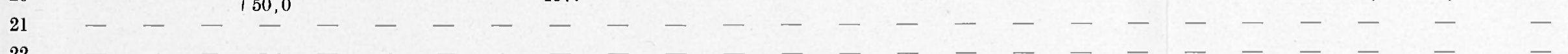

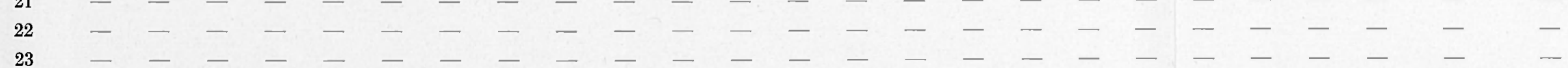

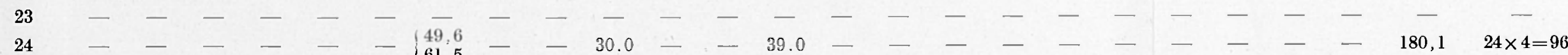

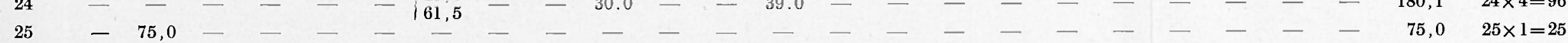

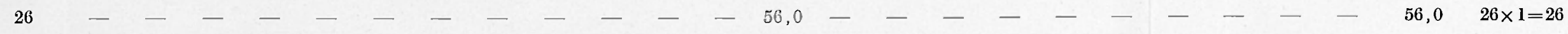

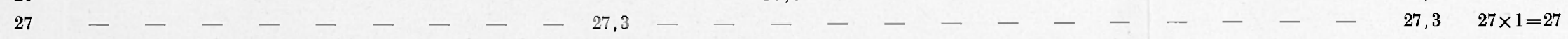

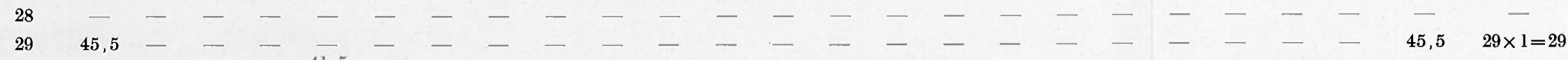

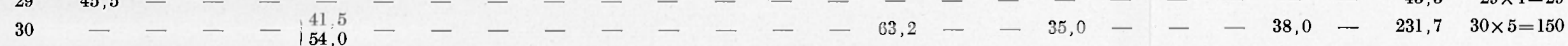

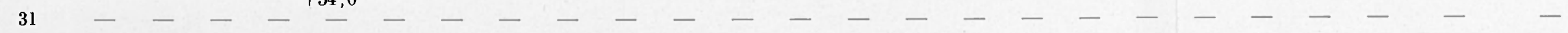

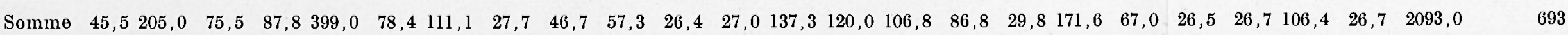

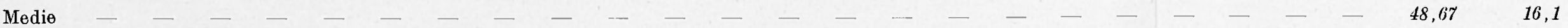

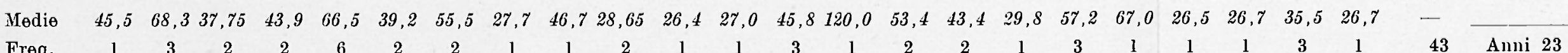

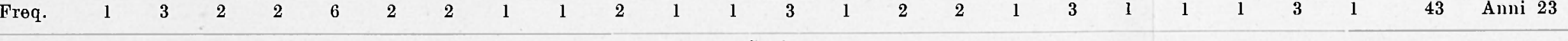




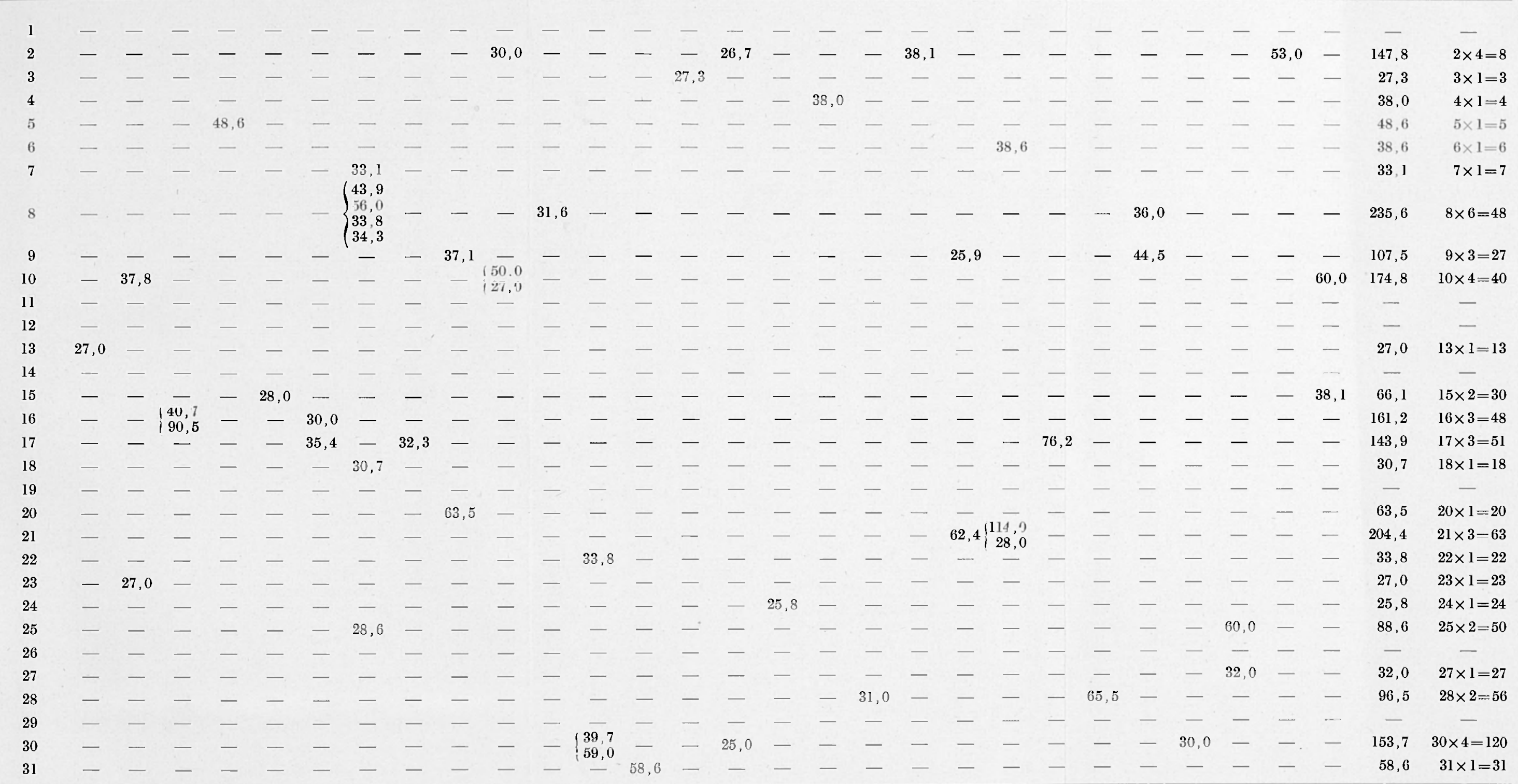

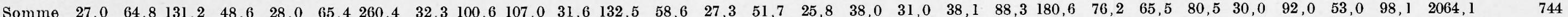




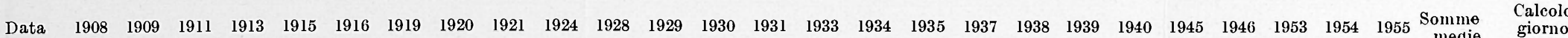
medie giorno

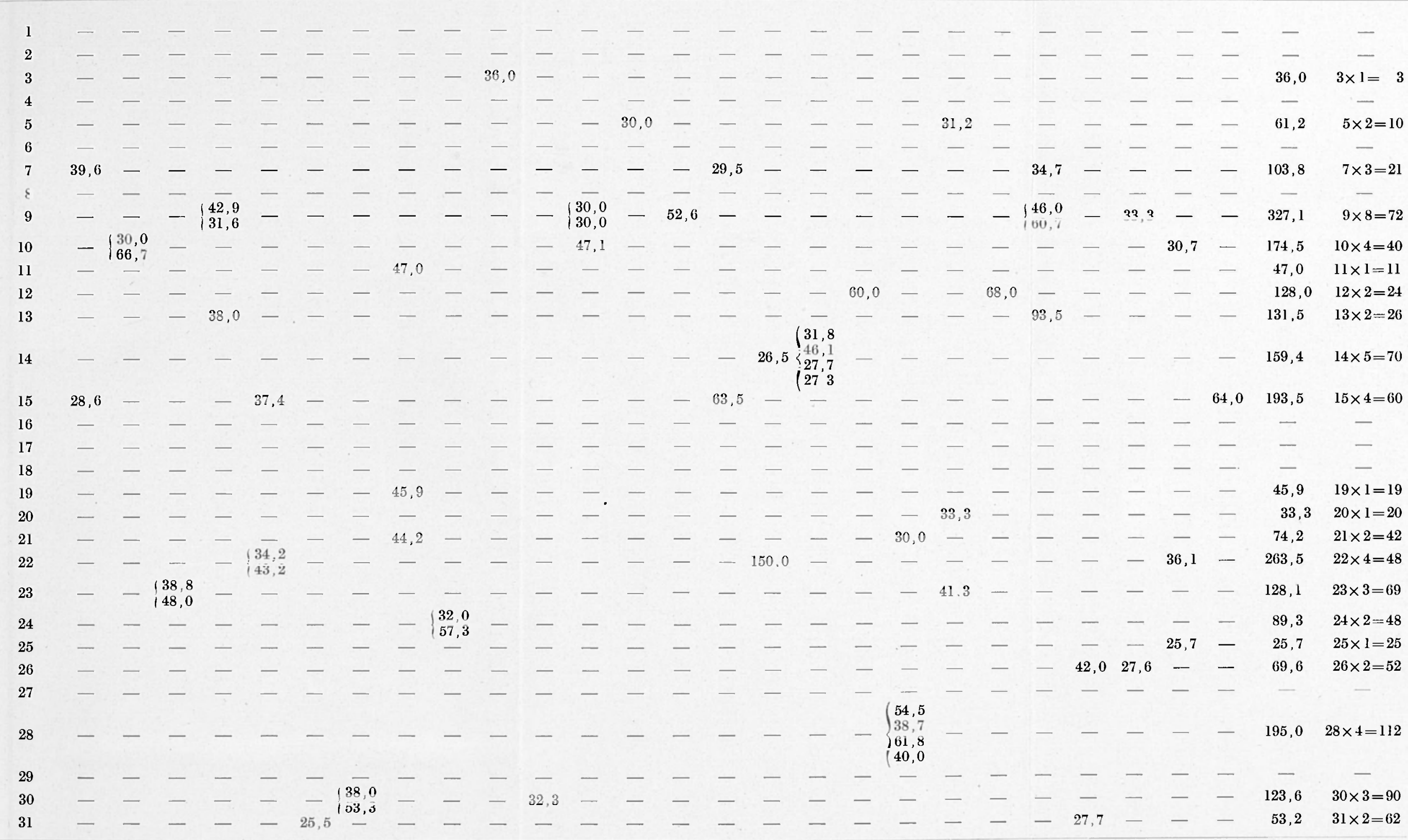

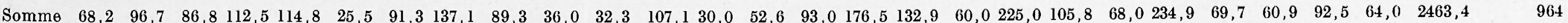

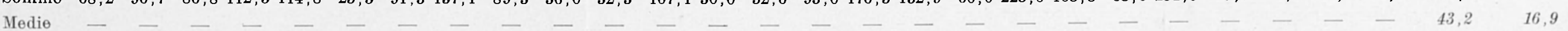

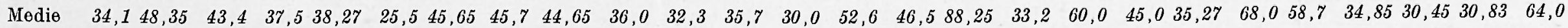

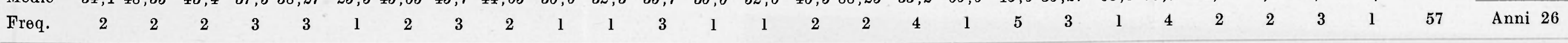




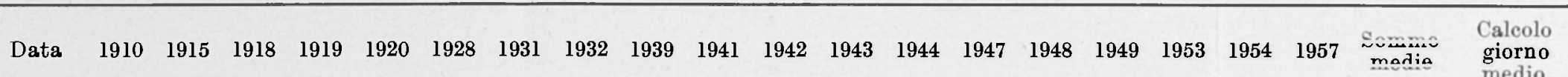

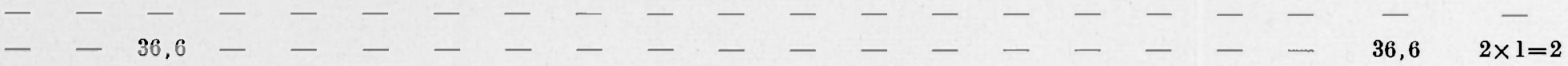

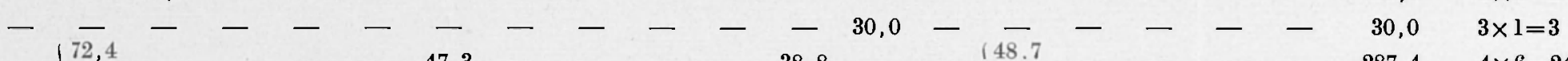

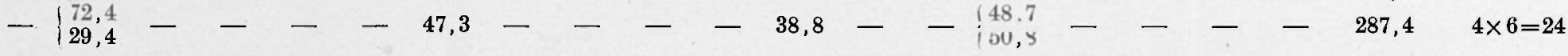

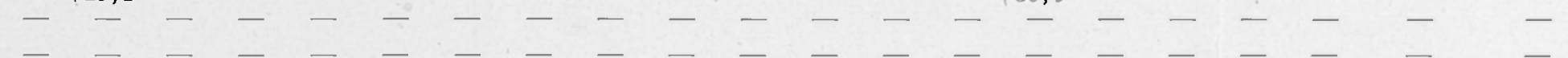

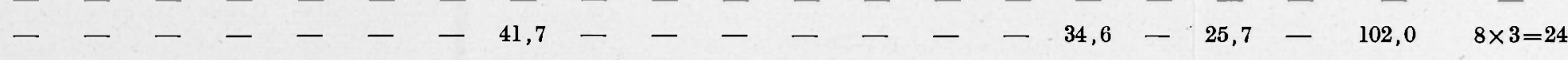

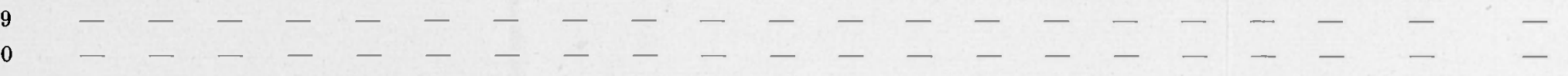

$$
\begin{aligned}
& 12-26,5-\quad-\left\{\begin{array}{l}
132,0 \\
40,0 \\
27,1 \\
39,1 \\
33,2
\end{array}\right.
\end{aligned}
$$

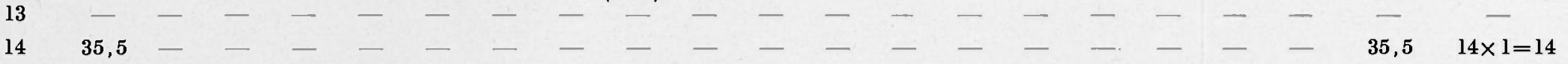

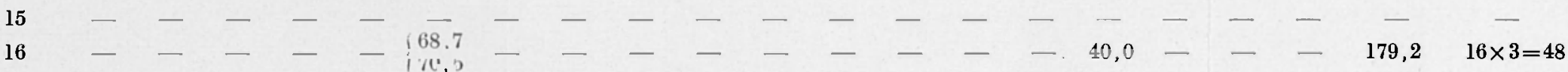

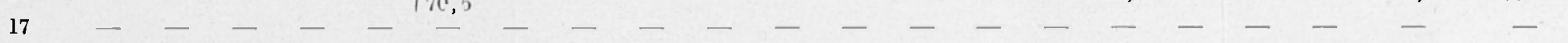

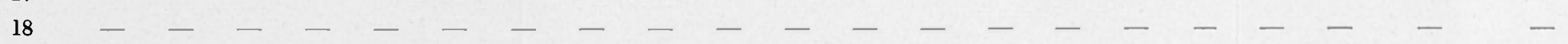

$$
\begin{aligned}
& 19-6-31,9-6-6-6-19 \times 1=19
\end{aligned}
$$

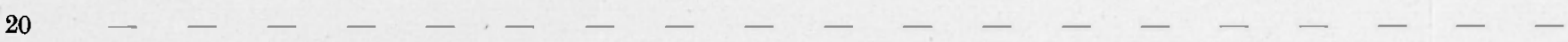

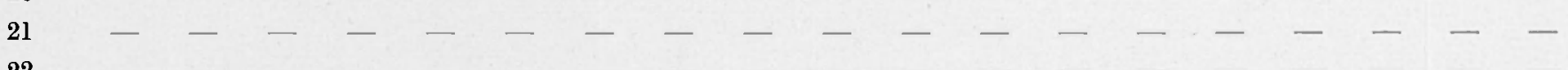

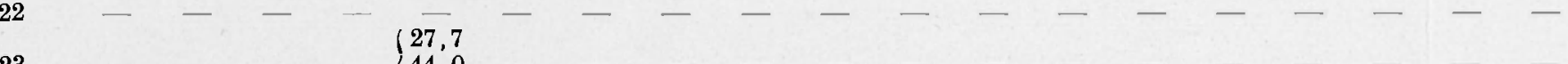

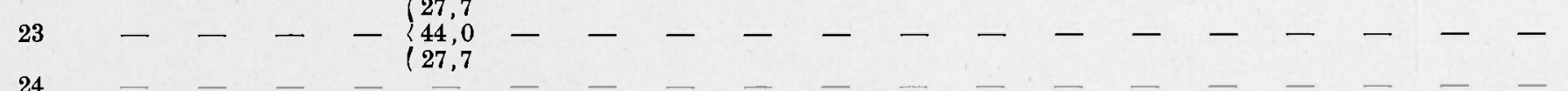

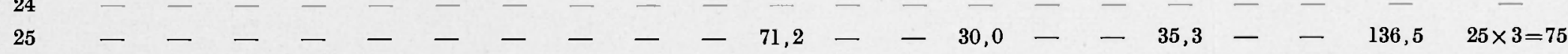

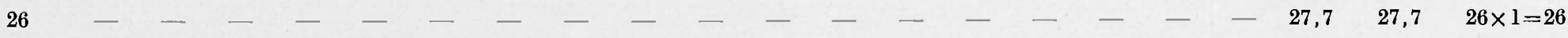

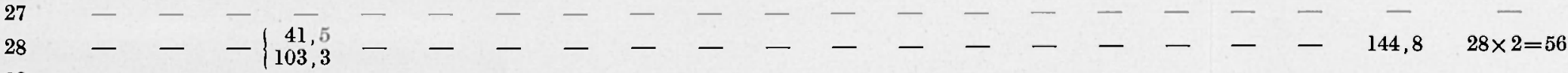

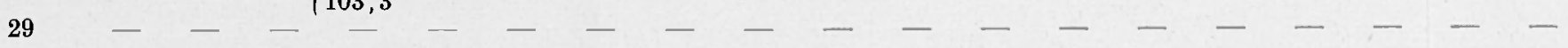

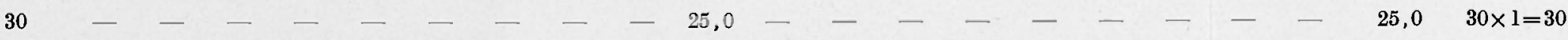$$
11
$$

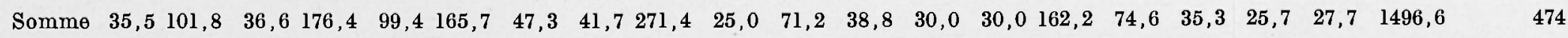

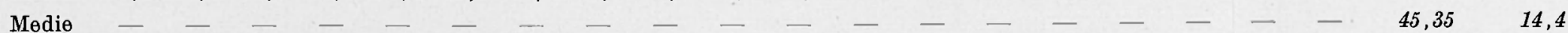
$\begin{array}{lccccccccccccccccccccccccc}\text { Medie } & 35,5 & 50,9 & 36,6 & 58,9 & 33,13 & 55,23 & 47,3 & 41,7 & 54,3 & 25,0 & 71,2 & 38,8 & 30,0 & 30,0 & 54,07 & 37,3 & 35,3 & 25,7 & 27,7 & - & -1 & \\ \text { Freq. } & 1 & 2 & 1 & 3 & 3 & 3 & 1 & 1 & 5 & 1 & 1 & 1 & 1 & 3 & 2 & 1 & 1 & 1 & 1 & 33 & \text { Anni } & 19\end{array}$ Mese $\quad S \quad \theta \quad t \quad t \quad \theta \quad m \quad b \quad r \quad \theta$ 
PRECIPITAZIONi DI NOTEVOLI INTENSITÀ A PAVIA DAL 1908 AL 1957

G. PANNOCCHIA

Seguito Tab. 11 (g)

\begin{tabular}{|c|c|c|c|c|c|c|c|c|c|c|c|}
\hline Data & 1913 & 1926 & 1929 & 1935 & 1939 & 1940 & 1941 & 1944 & 1953 & 1956 & $\begin{array}{l}\text { Domnine } \\
\text { medie }\end{array}$ \\
\hline
\end{tabular}

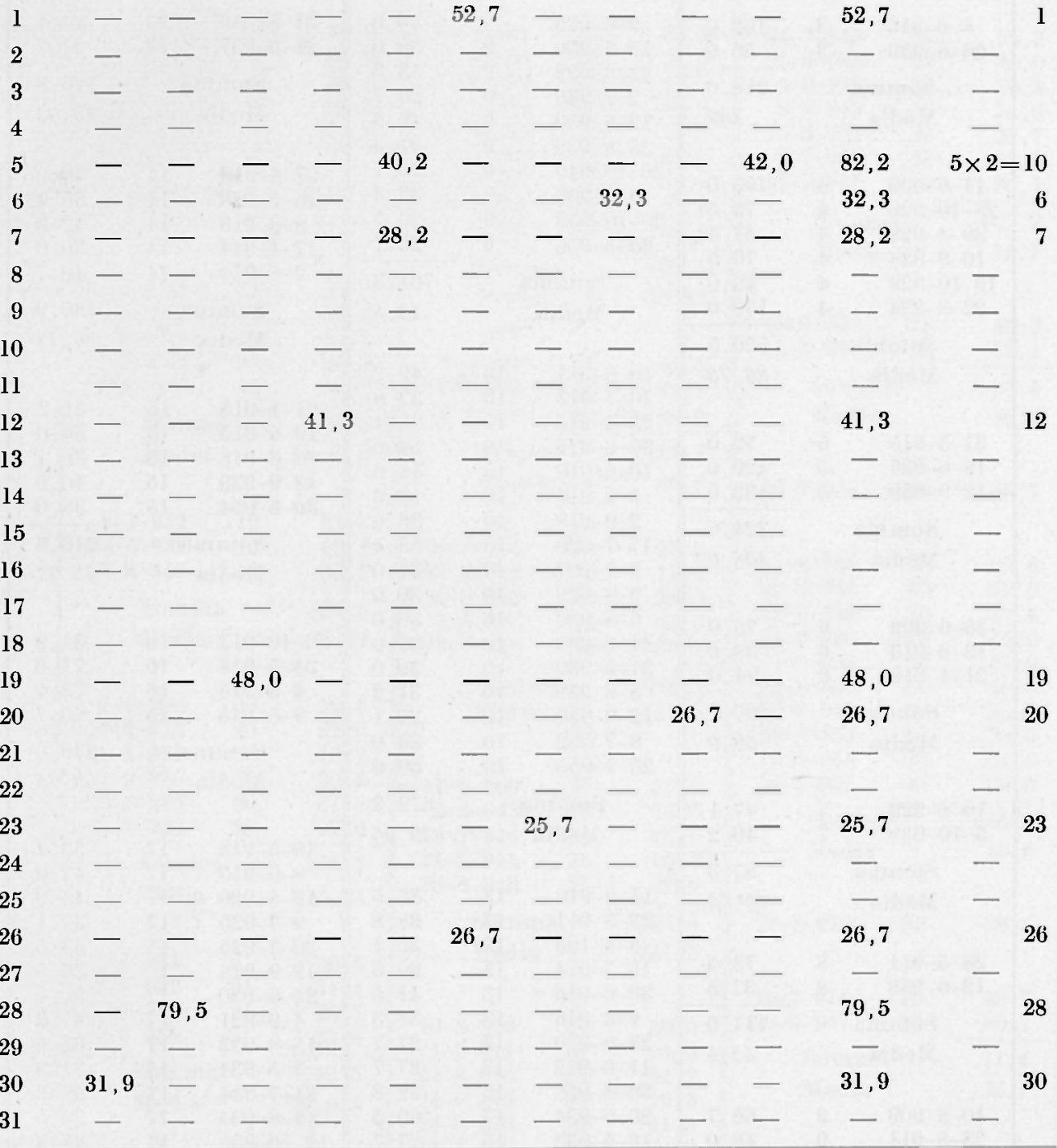

$\begin{array}{llllllllllllll}\text { Somme } & 31,9 & 79,5 & 48,0 & 41,3 & 68,4 & 79,4 & 25,7 & 32,3 & 26,7 & 42,0 & 475,2 & 182\end{array}$ Medie - _ - _ - - - - - - $\begin{array}{llllllllllll}\text { Medie } & 31,9 & 79,5 & 48,0 & 41,3 & 34,2 & 39,7 & 25,7 & 32,3 & 26,7 & 42,0 & -\end{array}$ $\begin{array}{llllllllllllllll}\text { Freq. } & 1 & 1 & 1 & 1 & 2 & 2 & 1 & 1 & 1 & 1 & 12 & \text { Anni } 10\end{array}$ 
PRECIPITAZIONI DI NOTEVOLI INTENSITÀ A PAVIA DAL 1908 AL 1957

(a)

Tabella III

G. PAN NOCCHIA

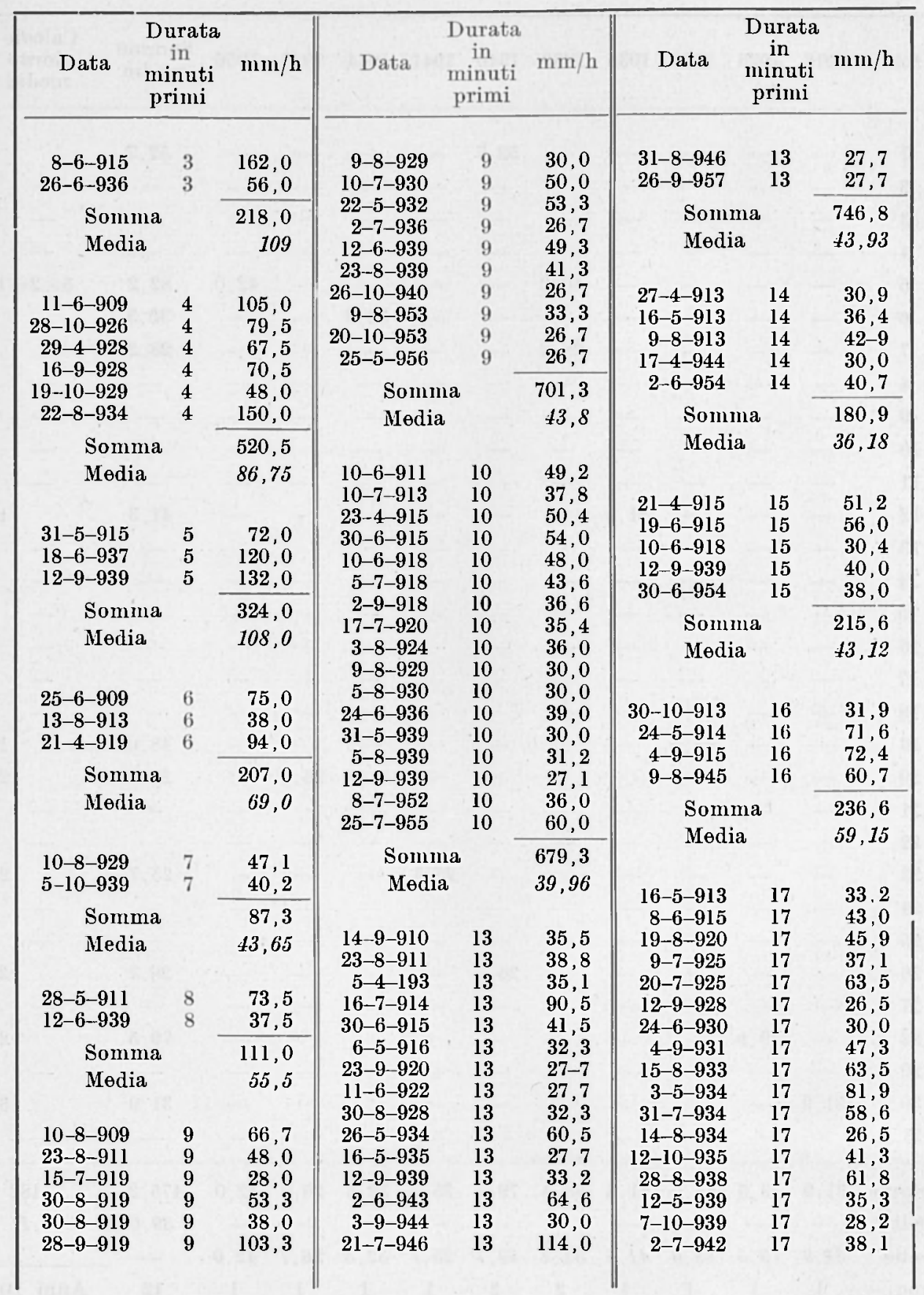




\begin{tabular}{|c|c|c|c|c|c|c|c|c|}
\hline \multicolumn{3}{|c|}{$\begin{array}{l}\text { Durata } \\
\text { in } \\
\text { minuti } \\
\text { primi }\end{array}$} & \multicolumn{3}{|c|}{$\begin{array}{c}\text { Durata } \\
\text { in } \\
\text { minuti } \\
\text { primi }\end{array}$} & \multicolumn{3}{|c|}{$\begin{array}{c}\text { Durata } \\
\text { in } \\
\text { minuti } \\
\text { primi }\end{array}$} \\
\hline $4-9-943$ & 17 & 38,8 & $24-8-921$ & 22 & 57,3 & $24-7-938$ & 26 & 25,8 \\
\hline $17-6-144$ & 17 & 67,0 & $7-5-925$ & 22 & 27,3 & $21-8-938$ & 26 & 30,0 \\
\hline $26-5-945$ & 17 & 37 , & $27-6-930$ & 22 & 27,3 & $4-9-948$ & 26 & 50,8 \\
\hline $13-8-945$ & 17 & 93 & $3-7-935$ & 22 & 27,3 & $8-9-949$ & 26 & 34,6 \\
\hline $17-6-950$ & 17 & 26,5 & $25-8-935$ & 22 & 27,3 & $15-6-954$ & 26 & 27,7 \\
\hline $25-9-953$ & 17 & 35,3 & $2-6-936$ & 22 & 42,3 & 2-7-956 & 26 & 53,0 \\
\hline \multirow{3}{*}{\multicolumn{2}{|c|}{$\begin{array}{l}\text { Somma } \\
\text { Media }\end{array}$}} & 1059,8 & 17-5-938 & 22 & 52,3 & $15-7-957$ & 26 & 38,1 \\
\hline & & 46,08 & $\begin{array}{l}13-6-938 \\
28-8-938\end{array}$ & $\begin{array}{l}22 \\
22\end{array}$ & $\begin{array}{l}43,6 \\
54,5\end{array}$ & \multirow{2}{*}{\multicolumn{2}{|c|}{$\begin{array}{l}\text { Somma } \\
\text { Media }\end{array}$}} & 607,2 \\
\hline & & & \multirow{3}{*}{\multicolumn{2}{|c|}{$\begin{array}{l}\text { Somma } \\
\text { Modia }\end{array}$}} & 494,1 & & & 35,7 \\
\hline $20-8-939$ & 18 & 33,3 & & & 38,01 & & & \\
\hline $1-10-940$ & 18 & 52,7 & & & & $13-5-935$ & 28 & 49,3 \\
\hline $6-10-944$ & 18 & 32,3 & \multirow{4}{*}{$\begin{array}{r}11-8-920 \\
8-9-932 \\
12-8-937\end{array}$} & \multirow{4}{*}{$\begin{array}{l}23 \\
23 \\
23\end{array}$} & & $11-5-955$ & 28 & 32,1 \\
\hline $21-5-948$ & 18 & 60,0 & & & $\begin{array}{l}47,0 \\
417\end{array}$ & \multirow{3}{*}{\multicolumn{2}{|c|}{$\begin{array}{l}\text { Somma } \\
\text { Media }\end{array}$}} & 81,4 \\
\hline $20-6-955$ & & 26,7 & & & $\begin{array}{l}41,7 \\
60,0\end{array}$ & & & 40,7 \\
\hline \multirow{2}{*}{\multicolumn{2}{|c|}{$\begin{array}{l}\text { Somma } \\
\text { Media }\end{array}$}} & 204,0 & & & & \multirow[b]{2}{*}{$16-9-928$} & & \multirow[b]{2}{*}{68,7} \\
\hline & & 40,8 & \multicolumn{2}{|c|}{$\begin{array}{l}\text { Somma } \\
\text { Media }\end{array}$} & $\begin{array}{r}148,7 \\
49,6\end{array}$ & & 29 & \\
\hline $8-7-932$ & 19 & 31,6 & & & & \multirow{10}{*}{$\begin{array}{r}15-8-908 \\
20-6-913 \\
13-8-915 \\
23-9-920 \\
1-6-933 \\
28-8-938 \\
30-9-941 \\
30-7-953 \\
15-8-955 \\
10-7-957\end{array}$} & & \\
\hline $30-6-938$ & 19 & 63,2 & \multirow{4}{*}{$\begin{array}{r}30-7-936 \\
25-9-942 \\
30-6-943 \\
9-8-945 \\
28-7-950\end{array}$} & \multirow{4}{*}{$\begin{array}{l}24 \\
24 \\
24 \\
24 \\
24\end{array}$} & 25,0 & & \multirow{9}{*}{$\begin{array}{l}30 \\
30 \\
30 \\
30 \\
30 \\
30 \\
30 \\
30 \\
30 \\
30\end{array}$} & \multirow{9}{*}{$\begin{array}{l}28,6 \\
50,0 \\
37,4 \\
44,0 \\
27,0 \\
40,0 \\
25,0 \\
30,0 \\
64,0 \\
60,0 \\
\end{array}$} \\
\hline $7-8-945$ & 19 & 34,7 & & & 71,2 & & & \\
\hline Somı & & 129,5 & & & $\begin{array}{l}35,0 \\
46,0\end{array}$ & & & \\
\hline Medi & & 43,17 & & & $\begin{array}{l}46,0 \\
65,5\end{array}$ & & & \\
\hline & & & \multirow{3}{*}{\multicolumn{2}{|c|}{$\begin{array}{l}\text { Somma } \\
\text { Media }\end{array}$}} & 242,7 & & & \\
\hline $7-8-908$ & 20 & 39,6 & & & 48.54 & & & \\
\hline $23-7-913$ & 20 & 27,0 & & & & & & \\
\hline $22-8$ & 20 & 43,2 & \multirow{5}{*}{$\begin{array}{r}11-6-909 \\
2-6-943 \\
9-7-944 \\
21-7-944 \\
26-8-946\end{array}$} & \multirow{5}{*}{$\begin{array}{l}25 \\
25 \\
25 \\
25 \\
25\end{array}$} & & & & \\
\hline 4015 & 20 & 29,4 & & & 25,0 & & & \\
\hline 24-6-919 & 20 & 61,5 & & & 72,0 & \multirow{3}{*}{\multicolumn{2}{|c|}{$\begin{array}{l}\text { Somma } \\
\text { Media }\end{array}$}} & \multirow{3}{*}{$\begin{array}{r}406,0 \\
40,6\end{array}$} \\
\hline $10-7-930$ & 20 & 27,0 & & & 25,9 & & & \\
\hline Somr & & 227,7 & & & $\begin{array}{l}62,4 \\
42,0\end{array}$ & & & \\
\hline & & & Somı & & 227,3 & $28-8-938$ & 31 & 38,7 \\
\hline $9-8-931$ & & 52,6 & Medi & & 45,46 & & & \\
\hline $2-5-932$ & 21 & 31,4 & & & & $16-7-914$ & 33 & 40,7 \\
\hline $23-10-941$ & 21 & 25,7 & $10-8-909$ & 26 & 30,0 & $20-5-947$ & 33 & 30,7 \\
\hline-946 & 21 & 38,6 & $1-6-911$ & 26 & 26,3 & Somı & & 71,4 \\
\hline Somr & & 148,3 & $22-8-915$ & 26 & 34,2 & Medi & & 35,7 \\
\hline Medi & & 37,075 & 24-6-919 & 26 & $\begin{array}{l}49,5 \\
415\end{array}$ & & & \\
\hline & & & $\begin{array}{l}28-9-919 \\
16-7-920\end{array}$ & 26 & 30,0 & $9-7-952$ & 34 & 44,5 \\
\hline $19-6-915$ & & 42,5 & $17-7-924$ & 26 & 32,3 & $5-10-956$ & 34 & 42,0 \\
\hline $19-9-919$ & 22 & 31,9 & $7-8-933$ & 26 & 29,5 & & & 86.5 \\
\hline $31-3-920$ & 22 & 31,9 & $14-8-935$ & 26 & 27,7 & & & 4325 \\
\hline 25-7-921 & 22 & 28,6 & $14-8-935$ & 26 & 46,1 & Medic & & \\
\hline
\end{tabular}


PRECIPITAZIONi Di NOTEVOLI INTENSITÁ A PAVIA DAL 1908 AL 1957

Seguito Tab. III (c)

G. PANNOCCHIA

\begin{tabular}{|c|c|c|c|c|c|c|c|c|}
\hline \multicolumn{3}{|c|}{$\begin{array}{c}\text { Durata } \\
\text { in } \\
\text { minuti } \\
\text { primi }\end{array}$} & \multicolumn{3}{|c|}{$\begin{array}{l}\text { Durata } \\
\text { in } \\
\text { minuti } \\
\text { primi }\end{array}$} & \multicolumn{3}{|c|}{$\begin{array}{c}\text { Durata } \\
\text { in } \\
\text { minuti } \\
\text { primi }\end{array}$} \\
\hline 5-7-917 & 35 & 24,9 & $13-7-911$ & 40 & 27,0 & $24-8-921$ & 45 & 32,0 \\
\hline $22-4$ & 35 & 30,0 & $31-8-916$ & 40 & 25,5 & 16-9-949 & 45 & 40,0 \\
\hline $12-8-940$ & 35 & 68 & \multirow{2}{*}{\multicolumn{2}{|c|}{$\begin{array}{l}\text { Somma } \\
\text { Media }\end{array}$}} & 52,5 & $\begin{array}{l}8-6-9 \\
27-7\end{array}$ & $\begin{array}{l}45 \\
45\end{array}$ & $\begin{array}{l}26,7 \\
320\end{array}$ \\
\hline $8-9-954$ & & & & & 26,25 & \multirow{2}{*}{\multicolumn{2}{|c|}{$\begin{array}{l}\text { Somma } \\
\text { Media }\end{array}$}} & \\
\hline \multicolumn{2}{|c|}{$\begin{array}{l}\text { Somma } \\
\text { Media }\end{array}$} & $\begin{array}{l}148,6 \\
37,15\end{array}$ & & & & & & $\begin{array}{r}130,6 \\
32,675\end{array}$ \\
\hline $99-6-999$ & & & $\begin{array}{r}8-7-921 \\
30-5-952\end{array}$ & $\begin{array}{l}41 \\
41\end{array}$ & $\begin{array}{l}43,9 \\
75,5\end{array}$ & 4-9-948 & 48 & 48,7 \\
\hline $\begin{array}{r}29-0-929 \\
2-7-930\end{array}$ & $\begin{array}{l}50 \\
36\end{array}$ & $\begin{array}{l}40,7 \\
30,0\end{array}$ & \multirow{2}{*}{\multicolumn{2}{|c|}{$\begin{array}{l}\text { Somma } \\
\text { Modia }\end{array}$}} & 119,4 & $\begin{array}{r}7-7-921 \\
20-6-913\end{array}$ & $\begin{array}{l}49 \\
50\end{array}$ & $\begin{array}{l}33,1 \\
37,8\end{array}$ \\
\hline $\begin{array}{r}30-7-933 \\
5-6-941\end{array}$ & $\begin{array}{l}36 \\
36\end{array}$ & $\begin{array}{l}59,0 \\
29,8\end{array}$ & & & 59,7 & $\begin{array}{l}12-9-939 \\
12-9-948\end{array}$ & $\begin{array}{l}56 \\
59\end{array}$ & 39,1 \\
\hline \multirow{2}{*}{\multicolumn{2}{|c|}{$\begin{array}{l}\text { Somma } \\
\text { Media }\end{array}$}} & 165,5 & & & & $14-8-935$ & 83 & 31,8 \\
\hline & & 41,375 & $\begin{array}{r}8-7-921 \\
16-6-932\end{array}$ & $\begin{array}{l}42 \\
42\end{array}$ & $\begin{array}{l}56,0 \\
26,4\end{array}$ & $\begin{array}{l}17-5-938 \\
22-8-954\end{array}$ & $\begin{array}{l}87 \\
56\end{array}$ & $\begin{array}{l}27,0 \\
36,1\end{array}$ \\
\hline $\begin{array}{r}9-8-913 \\
21-8-920 \\
26-8-953\end{array}$ & $\begin{array}{l}38 \\
38 \\
38\end{array}$ & $\begin{array}{l}31,6 \\
44,2 \\
27,6 \\
\end{array}$ & $\begin{array}{l}\text { Som } \\
\text { Med }\end{array}$ & & $\begin{array}{l}82,4 \\
41,2\end{array}$ & \multicolumn{2}{|c|}{$\begin{array}{l}\text { Somma } \\
\text { Media }\end{array}$} & $\begin{array}{l}316,3 \\
39,54\end{array}$ \\
\hline \multicolumn{2}{|c|}{$\begin{array}{l}\text { Somma } \\
\text { Media }\end{array}$} & $\begin{array}{l}103,4 \\
34,47\end{array}$ & $\begin{array}{l}25-8-911 \\
18-7-921 \\
22-7-933\end{array}$ & $\begin{array}{l}43 \\
43 \\
43\end{array}$ & $\begin{array}{l}35,9 \\
30,7 \\
33,8\end{array}$ & $\begin{array}{r}3-5-931 \\
13-4-937 \\
28-7-941 \\
17-7-949\end{array}$ & $\begin{array}{l}60 \\
60 \\
60 \\
60\end{array}$ & $\begin{array}{l}26,0 \\
26,5 \\
31,0 \\
76,2 \\
\end{array}$ \\
\hline $\begin{array}{r}23-9-920 \\
8-7-921\end{array}$ & $\begin{array}{l}39 \\
39\end{array}$ & $\begin{array}{l}27,7 \\
33,8\end{array}$ & $\begin{array}{l}25-9-947 \\
10-8-954\end{array}$ & $\begin{array}{l}43 \\
43\end{array}$ & $\begin{array}{l}30,0 \\
30,7\end{array}$ & \multicolumn{2}{|c|}{$\begin{array}{l}\text { Somma } \\
\text { Media }\end{array}$} & $\begin{array}{r}160,7 \\
40,175\end{array}$ \\
\hline \multicolumn{2}{|c|}{$\begin{array}{l}\text { Somma } \\
\text { Media }\end{array}$} & $\begin{array}{r}61,5 \\
30,75\end{array}$ & \multicolumn{2}{|c|}{$\begin{array}{l}\text { Somma } \\
\text { Media }\end{array}$} & $\begin{array}{l}161,1 \\
32,22\end{array}$ & $8-7-921$ & 162 & 34,3 \\
\hline
\end{tabular}

